ARTICLE

\title{
GLUT1 inhibition blocks growth of RB1-positive triple negative breast cancer
}

\author{
Qin Wu'1,2,3,14, Wail ba-alawi (10 2,34, Genevieve Deblois², Jennifer Cruickshank ${ }^{4}$, Shili Duan²,
} Evelyne Lima-Fernandes ${ }^{1,2}$, Jillian Haight ${ }^{4}$, Seyed Ali Madani Tonekaboni ${ }^{2,3}$, Anne-Marie Fortier (i) ${ }^{5}$, Hellen Kuasne ${ }^{5}$, Trevor D. McKee (1) 2,6, Hassan Mahmoud 2,3,7, Michelle Kushida ${ }^{8}$, Sarina Cameron ${ }^{2,3}$, Nergiz Dogan-Artun ${ }^{2,3}$, WenJun Chen', Yan Nie ${ }^{1,2,3}$, Lan Xin Zhang ${ }^{1}$, Ravi N. Vellanki², Stanley Zhou (1) 2,3, Panagiotis Prinos (1) ${ }^{1}$, Bradly G. Wouters ${ }^{2,3}$, Peter B. Dirks (10) 8,9,10, Susan J. Done (D) ${ }^{4}$, Morag Park (i) ${ }^{5}$, David W. Cescon (1) 4, Benjamin Haibe-Kains 2,3,11,12,13, Mathieu Lupien (1) 2,3,12凶 \& Cheryl H. Arrowsmith (1) 1,2,3凶

Triple negative breast cancer (TNBC) is a deadly form of breast cancer due to the development of resistance to chemotherapy affecting over $30 \%$ of patients. New therapeutics and companion biomarkers are urgently needed. Recognizing the elevated expression of glucose transporter 1 (GLUT1, encoded by SLC2A1) and associated metabolic dependencies in TNBC, we investigated the vulnerability of TNBC cell lines and patient-derived samples to GLUT1 inhibition. We report that genetic or pharmacological inhibition of GLUT1 with BAY-876 impairs the growth of a subset of TNBC cells displaying high glycolytic and lower oxidative phosphorylation (OXPHOS) rates. Pathway enrichment analysis of gene expression data suggests that the functionality of the E2F pathway may reflect to some extent OXPHOS activity. Furthermore, the protein levels of retinoblastoma tumor suppressor (RB1) strongly correlate with the degree of sensitivity to GLUT1 inhibition in TNBC, where RB1-negative cells are insensitive to GLUT1 inhibition. Collectively, our results highlight a strong and targetable RB1-GLUT1 metabolic axis in TNBC and warrant clinical evaluation of GLUT1 inhibition in TNBC patients stratified according to RB1 protein expression levels.

\footnotetext{
${ }^{1}$ Structural Genomics Consortium, University of Toronto, Toronto, ON M5G 1L7, Canada. ${ }^{2}$ Princess Margaret Cancer Centre, University Health Network, Toronto M5G 1 L7 ON, Canada. ${ }^{3}$ Department of Medical Biophysics, University of Toronto, Toronto M5G 2M9 ON, Canada. ${ }^{4}$ The Campbell Family Institute for Breast Cancer Research, Princess Margaret Cancer Centre, Toronto, ON M5G 2M9, Canada. ${ }^{5}$ Goodman Cancer Research Centre, McGill University, Montreal, QC H3A 1A3, Canada. ${ }^{6}$ Princess Margaret Cancer Centre, STTARR Innovation Facility, Toronto, ON, Canada. ${ }^{7}$ Faculty of Computer and Informatics, Benha University, Benha, Egypt. ${ }^{8}$ Developmental and Stem Cell Biology Program and Arthur and Sonia Labatt Brain tumor Research Centre, The Hospital for Sick Children, Toronto, ON M5G 0A4, Canada. ${ }^{9}$ Division of Neurosurgery, University of Toronto, Toronto, ON M5S 1A8, Canada.

${ }^{10}$ Departments of Molecular Genetics and Surgery, University of Toronto, Toronto, ON M5S1A8, Canada. ${ }^{11}$ Department of Computer Science, University of Toronto, Toronto M5T 3A1 ON, Canada. ${ }^{12}$ Ontario Institue for Cancer Research, Toronto M5G 2M9 ON, Canada. ${ }^{13}$ Vector Institute for Artificial Intelligence, Toronto, ON, Canada. ${ }^{14}$ These authors contributed equally: Qin Wu, Wail ba-alawi. ${ }^{凶}$ email: Mathieu.Lupien@uhnresearch.ca; Cheryl.Arrowsmith@uhnresearch.ca
} 
B reast cancer is the most common female cancer worldwide, with 1.7 million new cases and over 520,000 deaths recorded in $2012^{1}$. Triple negative breast cancer (TNBC) is a highly aggressive subtype of breast cancer that lacks the expression of the estrogen receptor a (ER $\alpha)$, progesterone receptors (PR) and the epidermal growth factor receptor 2 (HER2). TNBC represents $15-20 \%$ of breast cancer cases but accounts for $25 \%$ of deaths ${ }^{2}$. In addition, TNBC has a higher metastatic rate $(\sim 2.5$ fold) within 5 years of diagnosis and poorer overall survival rate (4.2 vs. 6 years) compared to receptor positive breast cancer subtypes ${ }^{3,4}$. This poor outcome derives from the heterogeneous nature of the disease, coupled with the lack of highly recurrent and/or actionable biomarkers that are informative for therapy ${ }^{2,5}$. Furthermore, while some TNBC are initially chemosensitive, $23 \%$ of patients recur within 5 years from diagnosis and over $30 \%$ develop drug resistance tumors ${ }^{6}$. Therefore, there is an urgent need to improve our understanding of the molecular basis for TNBC development and progression to discover effective therapeutic targets and their companion test to improve the outcome in patients.

Metabolic adaptation is inherent to tumorigenesis to meet the increased requirements for bioenergetic, biosynthetic, and detoxification demands of malignant cells ${ }^{7}$. An increased aerobic glycolysis rate is a common metabolic feature in many cancer cells, and has been under extensive investigation as a therapeutic focus in cancer ${ }^{8,9}$. Among breast cancers, TNBC cells have an elevated glycolytic gene signature and concomitant lower OXPHOS signature compared to other breast cancer subtypes such as the hormone-positive luminal breast cancer ${ }^{10}$. High expression of glucose transporter 1 (GLUT1), a key rate-limiting factor for glucose uptake, is significantly elevated in basal-like breast cancer subtype ${ }^{11}$ (the most common type of $\mathrm{TNBC}^{12}$ ). This suggests a key role for GLUT1 in regulating TNBC cell metabolism. Targeting GLUT1 with small molecules, such as STF-31, WZB-117 and BAY-876, has been investigated in various types of cancers with promising results ${ }^{13-15}$. GLUT1 inhibition, either by a short hairpin RNA (shRNA) or WZB-117 inhibitor treatment showed anti-proliferation effects in MDA-MB-231 and Hs 578T TNBC cell lines, again supporting GLUT1 as a possible target in $\mathrm{TNBC}^{16,17}$. Hence, a more systematic investigation and better understanding of the mechanisms regulating GLUT1 dependency is urgently needed to assess the benefits of pharmacological inhibition of GLUT1 for TNBC treatment in preclinical settings.

The inherent plasticity of cellular metabolism and the high degree of metabolic heterogeneity in TNBCs pose great challenges for metabolism targeting therapy ${ }^{18}$. Recent work suggests that heterogeneous metabolic dependencies within cancer cells underline the differential therapeutic vulnerabilities ${ }^{19}$. Therefore, in order for GLUT1 inhibition to be a successful strategy for TNBC therapy, the precise contexts in which this metabolic pathway is essential need to be identified. In addition to tumor microenvironment, metabolic dependencies can be driven by genetic lesions, such as myc amplification and Kras mutation ${ }^{20,21}$. However, this oncogene-driven cancer metabolism is incredibly complex and context-specific across cancer types ${ }^{21,22}$. Reliable biomarkers for predicting GLUT1 dependence and GLUT1 inhibition sensitivity are still lacking in TNBC.

In this study, we systematically assess the vulnerability of a wide range of well-characterized TNBC cell lines to GLUT1 pharmacological inhibition. We then identify the molecular basis underlying GLUT1 dependencies, and validate our results in patient-derived organoids and tumor explants. Finally, we identify RB1 protein levels as a predictive biomarker for GLUT1 sensitivity, which may potentially be used to stratify TNBC patients that would benefit from targeted GLUT1 therapy.

\section{Results}

Growth of a subset of TNBC relies on GLUT1 activity. To test the GLUT1 dependency of TNBC, we first investigated whether the expression level of $S L C 2 A 1$, the gene encoding GLUT1, was increased in TNBC by interrogating two large independent publicly available clinical cohorts, the TCGA and METABRIC ${ }^{23,24}$. In both cohorts examined, SLC2A1 mRNA expression is significantly elevated in basal-like subtype (corresponding to the most common subtype of $\mathrm{TNBC}^{11}$ ) compared to estrogen receptor positive and HER2-amplified breast tumors (TCGA: $p=3.33 \mathrm{e}-11$; METABRIC: $p=2.53 \mathrm{e}-8$ ) (Fig. 1a, b). Similarly, SLC2A1 elevated mRNA levels were observed in a smaller, independent breast cancer patient-derived xenograft (PDX) cohort from the Princess Margaret Cancer Center (PM-PDXs) (Fig. 1c. $p=1.67 \mathrm{e}-2$ ). PAM50based breast cancer subtype classification across these datasets also revealed increased SLC2A1 mRNA expression levels in the basallike subtype over all other subtypes (Supplementary Fig. 1a-c) ${ }^{25}$. Similarly, GLUT1 protein levels were found to be higher in TNBC compared to luminal breast tumors in the Clinical Proteomic Tumor Analysis Consortium (CPTAC) Confirmatory/Discovery dataset (Supplementary Fig. 1d) ${ }^{26}$.

To further assess the function of GLUT1 as a target and the feasibility of GLUT1 inhibition as a therapy for TNBC patients, we treated a panel of 17 TNBC cell lines with the small molecule GLUT1 inhibitor BAY-876. Among the reported GLUT inhibitors, BAY-876, is the only inhibitor that is both highly potent and selective for GLUT1 over other glucose transporters (Supplementary Table 1). Significant growth inhibitory effects were observed in 11 of the 17 TNBC cell lines (Fig. 1d and Supplementary Fig. 1e, f) based on a half maximal inhibitory concentration $\left(\mathrm{IC}_{50}\right)$ value of $<5 \mu \mathrm{M}$ (range from 0.1 to $4.5 \mu \mathrm{M}$ ). Although BAY-876 was not tested in normal mammary cells, its low toxicity can be extrapolated from its testings on several other normal cell lines (Supplementary Fig. 1g) and primary cells ${ }^{27,28}$. The $\mathrm{IC}_{50}$ value for BAY-876 was $>10 \mu \mathrm{M}$ for the six 'resistant' TNBC cell lines, the maximum dose used in the treatment. To complement the results from short-term treatments, we performed long-term (14 days) colony-forming assays to determine if the inhibitory effects of BAY-876 are sustained over time. At BAY-876 concentrations of $1 \mu \mathrm{M}$, proliferation of sensitive cell lines (HCC1806 and Hs 578T) was severely inhibited, whereas the resistant cell lines showed little effect (MDA-MB-436 and MDA-MB-468) (Supplementary Fig. 1h). These data confirm the heterogeneous response to GLUT1 pharmacologic inhibition across TNBC cell lines. We next evaluated the effect of siRNA-mediated silencing of GLUT1 on cell proliferation. Consistent with the results from pharmacological inhibition of GLUT1 with BAY-876, SLC2A1 silencing reduced GLUT1 protein levels (Fig. 1e) and significantly impaired the growth of TNBC cell lines sensitive to BAY-876 (HCC1806 and Hs 578T) but had no impact on the growth of BAY-876-resistant TNBC cell lines (MDA-MB-436 and MDA-MB-468) (Fig. 1f). In agreement, partial deprivation of glucose from the culture media selectively impaired the growth of cell lines sensitive to BAY-876 treatment but had no significant effect on the BAY-876-resistant cell lines over 5 days (Fig. $1 \mathrm{~g}$ ).

We next characterized the mechanism of BAY-876 impaired growth in TNBC cell lines by quantifying the impact on cell cycle and apoptosis. The BAY-876 sensitive HCC1806 and Hs 578T cell lines demonstrated a modest but significant decrease in the $S$ phase, with a concurrent increase in G1 phase with $3 \mu \mathrm{M}$ BAY-876 treatment or GLUT1 knockdown (Fig. 1h and Supplementary Fig. 1i, j). In contrast, MDA-MB-436 and MDA-MB-468 cells showed no significant changes in cell cycle progression (Fig. 1h). Moreover, caspase 3/7 staining showed a significant increase in the number of apoptotic cells in BAY-876 sensitive compared to resistant cell lines upon BAY-876 treatment or GLUT1 knockdown 

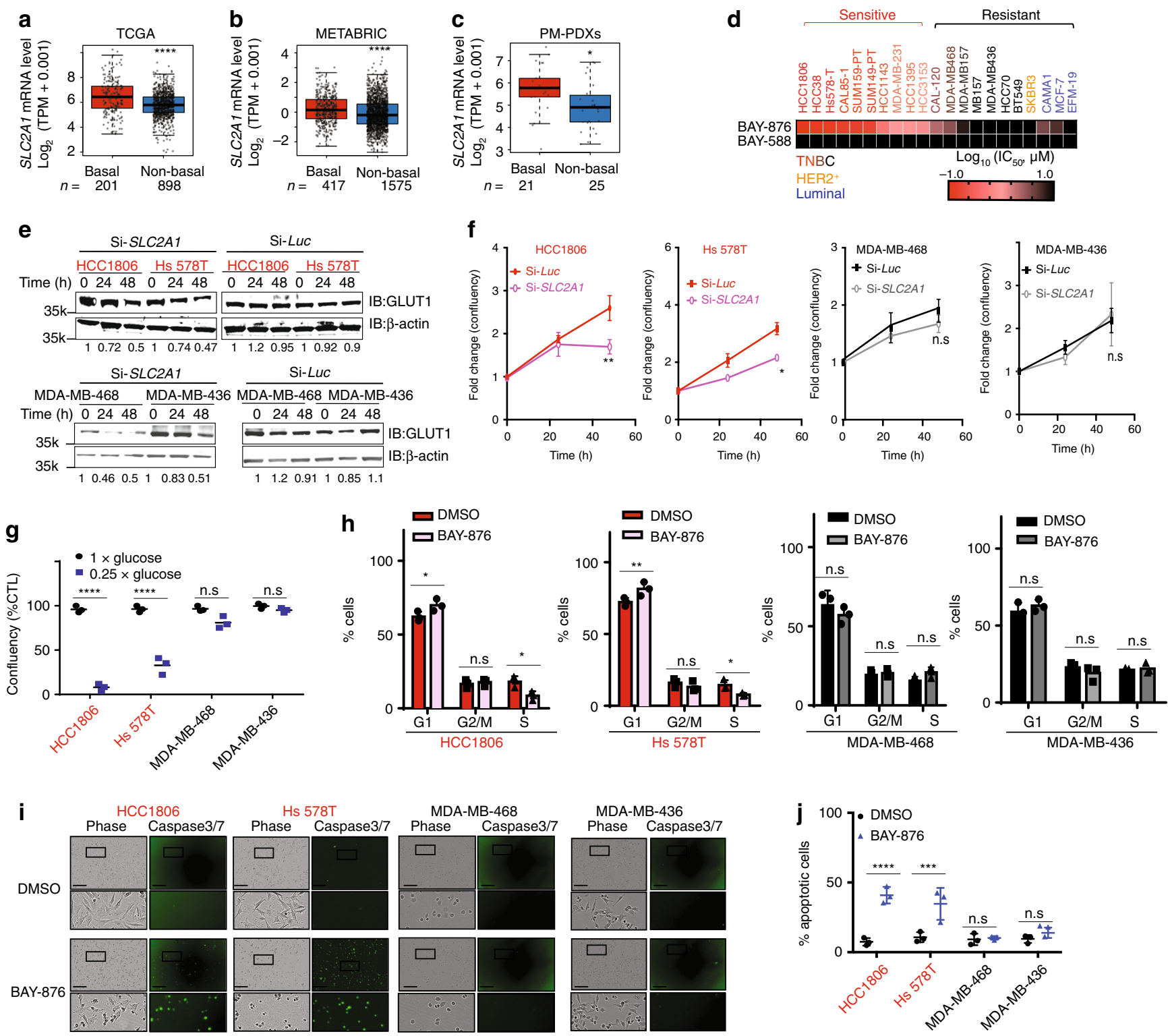

Fig. 1 Growth of a subset of TNBC relies on GLUT1 activity. SLC2A1 gene expression in the a TCGA breast cancer datasets, b METABRIC breast cancer datasets, and $\mathbf{c}$ Princess Margaret Hospital PDXs datasets (PM-PDXs). According to PAM50 classification, the cohorts were designated as basal and nonbasal subtypes. Gene expression is reported as $\log _{2}(T P M+0.001)$. The number of patients $(n)$ per group is indicated. Wilcoxon rank sum test. ${ }^{*} p<0.05$; ${ }^{\star \star \star \star \star} p<0.0001$. d Heatmap of mean $I C_{50}$ values for the indicated 21 breast cancer cell lines. $n=4$, mean \pm s.d. e Representative immunoblots showing the siRNA knockdown of GLUT1 or luciferase control in the BAY-876-sensitive lines (HCC1806 and Hs 578T) and BAY-876-resistant lines (MDA-MB-436 MDA-MB-468). Relative band intensities shown below blots. $\mathbf{f}$ Normalized cell confluency of GLUT1 knockdown cells or siRNA luciferase control cells for the indicated time post siRNA transduction. Cell confluency is normalized to T0 time point. $n=4$, mean \pm s.d. Two-way ANOVA. ${ }^{\star} p<0.05 ;{ }^{\star \star} p<0.01 ;$ n.s. not significant. $\mathbf{g}$ Cell growth of TNBC lines cultured in complete DMEM medium with or without glucose deprivation for 5 days. $n=4$, mean \pm s.d. Twoway ANOVA. ${ }^{\star \star \star \star} p<0.0001$. h Flow cytometry cell cycle analysis for indicated cells cultured with or without BAY-876 treated for 72 h. $n=3$, mean \pm s.d. Two-way ANOVA. ${ }^{\star} p<0.05 ;{ }^{\star \star} p<0.01 ;{ }^{\star \star \star} p<0.001$; n.s. not significant. i Representative images of caspase $3 / 7$ staining. The scale bar represents $300 \mu \mathrm{M}$. j Apoptotic cell counts of BAY-876 treated for 3 days by caspase $3 / 7$ staining. $n=3$, mean \pm s.d. Two-way ANOVA. ${ }^{\star \star \star} p<0.001 ;{ }^{* \star \star \star} p<0.0001$; n.s. not significant. Source data are provided as a Source Data file.

(Fig. 1i, j and Supplementary Fig. 1k). Taken together, these data showed that GLUT1 inhibition either by siRNA-mediated GLUT1 silencing or by pharmacological inhibition using BAY876 treatment, results in attenuated cell growth and proliferation, increased cell cycle arrest and increased cell apoptosis, which collectively contribute to growth suppression in a subset of TNBC cells.

OXPHOS levels correlate with the response to GLUT1 inhibition. As our data indicated that BAY-876 treatment selectively impairs the growth of a subset of TNBC cell lines, we assessed the mechanism conferring this heterogeneous response to GLUT1 inhibition. Because glucose is the fuel for glycolytic cellular metabolism, we reasoned that sensitivity to GLUT1 inhibition may be connected to the basal metabolic state of each cell line. Bioenergetic profiling revealed that the basal glycolytic rate as reflected by the extracellular acidification rate (ECAR) and mitochondrial oxygen consumption rates (OCR) indicative of oxidative phosphorylation (OXPHOS), discriminates between BAY-876 sensitive versus resistant TNBC cell lines (Fig. 2a). 
a

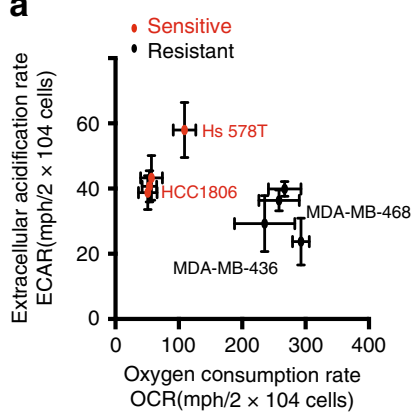

e

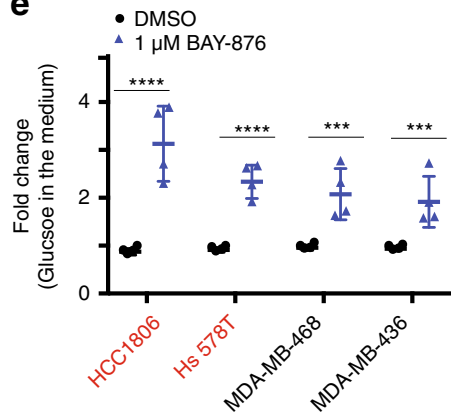

h

\section{Fold change}

(Glutamine in the media)

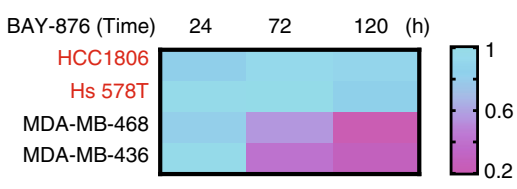

b

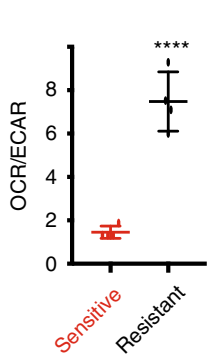

C

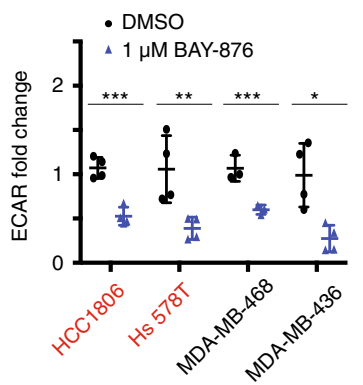

d

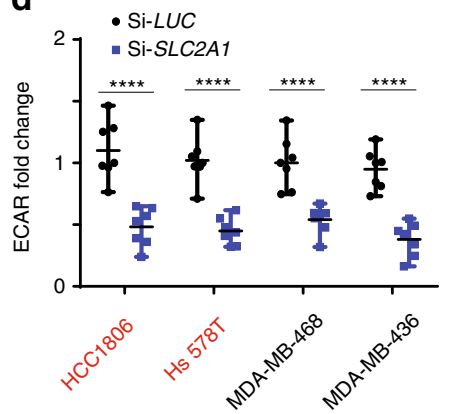

f

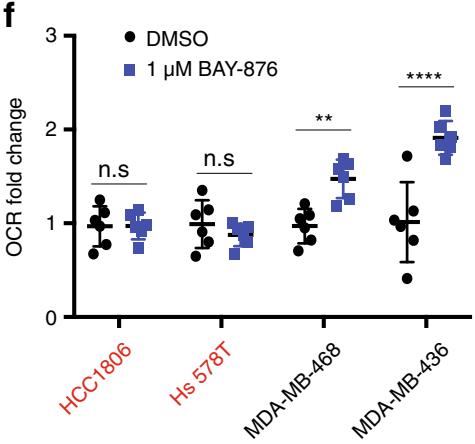

g

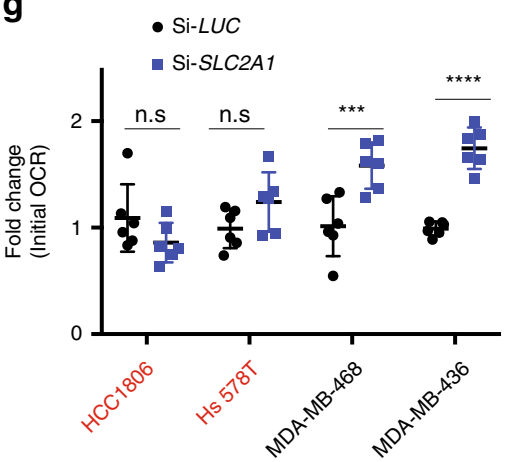

i

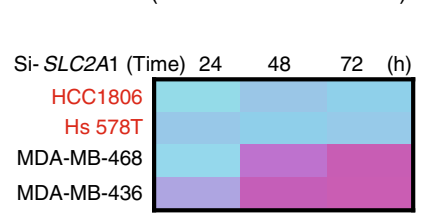

Fold change Fold change

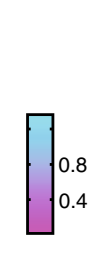

j

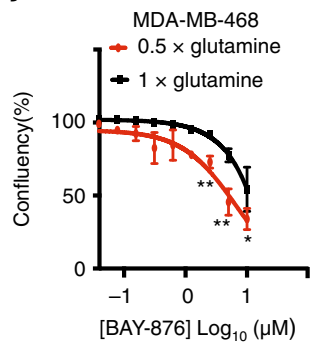

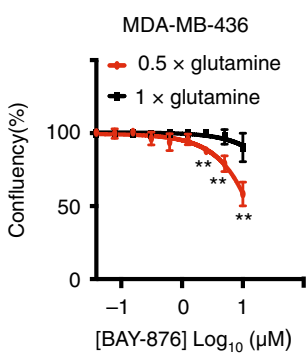

Fig. 2 OXPHOS levels correlate with the response to GLUT1 inhibition. a OCR and ECAR were measured for each of BAY-876-sensitive lines (red) and resistant lines (black). b OCR and ECAR ratio were calculated for each cell line. Student's $t$ test, ${ }^{\star \star \star \star \star} p<0.0001$. $\mathbf{c}$ ECAR values measurement of cells with or without BAY-876 treatment for 5 days. $n=4$; mean \pm s.d.; Two-way ANOVA. ${ }^{\star} p<0.05 ;{ }^{\star \star} p<0.01$; ${ }^{\star \star \star} p<0.001$. d ECAR values measurement of cells with siRNA of GLUT1 knockdown or luciferase control. $n=7$; mean \pm s.d. Two-way ANOVA. ${ }^{\star \star \star *} p<0.0001$. e Glucose uptake analysis were performed in cells following BAY-876 treatment for the 5 days. $n=4$; mean \pm s.d. Two-way ANOVA. ${ }^{\star \star \star} p<0.001 ;{ }^{\star \star \star \star} p<0.0001$; f A trace of OCR values from a mitochondrial stress test of cells with or without BAY-876 treatment for 5 days. $n=4$; mean \pm s.d. Two-way ANOVA. ${ }^{\star \star \star \star} p<0.0001 ; n . s$. not significant. g A trace of OCR values from a mitochondrial stress test of cells with siRNA of GLUT1 or luciferase control. $n=6$; mean \pm s.d. Two-way ANOVA. ${ }^{\star \star \star} p<$ $0.001 ;{ }^{* \star \star} p<0.0001 ; n$.s. not significant. $\mathbf{h}$ Glutamine uptake analysis was performed in cells following $1 \mu \mathrm{M} \mathrm{BAY}-876$ treatment. $n=3$. i Glutamine uptake analysis performed in cells transfected with $25 \mathrm{nM}$ siSLC2A1 (bottom). $n=2$. j Growth curves of MDA-MB-468 cells (left) and MDA-MB-436 cells (right) cultured in complete DMEM medium with or without glutamine deprivation treated with indicated nine doses of BAY-876 for 5 days. $n=3$; mean \pm s.d. Two-way ANOVA. ${ }^{\star \star} p<0.01 ;{ }^{\star \star \star} p<0.001 ;{ }^{\star \star \star \star} p<0.0001$. Source data are provided as a Source Data file.

Whereas resistant cell lines exhibited slightly decreased ECAR (glycolytic rates), they display a 3 -fold higher OCR (oxygen consumption rate) compared to sensitive cell lines at the basal level (in absence of BAY-876) (Fig. 2a). The ratio of OCR to ECAR (OCR/ECAR), indicative of higher reliance on OXPHOS, was significantly higher in resistant compared to sensitive TNBC cell lines (Fig. 2b). This observation indicates that BAY-876resistant cells display higher levels of OXPHOS at the basal state compared to BAY-876-sensitive TNBC cell lines.

In addition to the basal metabolic bioenergetic profile, a subclass of breast cancer cells have also been reported to switch from aerobic glycolysis to OXPHOS under limiting glucose conditions, as observed in cervical cancer, glioma, and pancreatic cancer cells ${ }^{29,30}$. This metabolic plasticity illustrates the interplay between glycolysis and OXPHOS, enabling the cells to adapt their bioenergetic profile to microenvironmental changes ${ }^{31}$. In agreement with BAY-876 inhibiting glucose uptake, the rate of glycolysis was significantly decreased upon GLUT1 inhibition, either by BAY-876 treatment or GLUT1 knockdown, as measured by decreased ECAR (Fig. 2c, d), glucose uptake (Fig. 2e), and lactate secretion (Supplementary Fig. 2a) in both BAY-876 sensitive and resistant TNBC cell lines. However, BAY-876resistant cell lines display approximately double OCR upon GLUT1 inhibition with BAY-876 treatment or GLUT1 knockdown, while no significant difference in OCR was observed in sensitive TNBC cell lines (Fig. 2f, g, Supplementary Fig. 2b-e). This suggests that BAY-876-resistant cells can adopt an increased OXPHOS metabolic profile to compensate for decreased glucose uptake, thereby enabling continued cell growth and cell survival. Since glutamine is utilized as a major energy source to drive OXPHOS, we next tested the dependence of both sensitive and resistant TNBC cell lines on glutamine. BAY-876-resistant TNBC 
cell lines exhibited glutamine depletion in the media (indicative of increased glutamine uptake) either upon SLC2A1 knockdown leading to depleted GLUT1 levels or BAY-876 treatment (Fig. 2h, i). In addition, removal of glutamine from the growth medium resulted in an increased sensitivity to BAY-876 in resistant TNBC cell lines, suggesting a strong dependence of resistant cells to glutamine-fueled OXPHOS to bypass growth suppression induced by GLUT1 inhibition (Fig. 2j). These results further support the ability of BAY-876-resistant TNBC cells to adapt their bioenergetic profile and metabolic requirements upon blocking GLUT1.

RB1 protein level discriminates response to GLUT1 inhibition. GLUT1 is known to influence a wide variety of biological processes, however, it is still unclear how these underlie the GLUT1 dependency of cancer cells ${ }^{32,33}$. To address this key question, we first examined molecular and phenotypic features that correlated with sensitivity or resistance to BAY-876 using the $\mathrm{IC}_{50}$ values calculated from our 17 TNBC cell lines (Fig. 1d). The highly reproducible responses identified in both sensitive and resistant cell lines, indicate that BAY-876 treatment and GLUT1 inhibition are not universally cytotoxic. To identify the molecular mechanism(s) for cellular drug resistance, we considered several candidate pathways. Since BAY-876 efficiently decreased glucose uptake and glycolysis rates in highly resistant cell lines (Fig. 2c, d), we ruled out drug-efflux pump or impaired drug metabolism mechanisms which could decrease the effective cellular concentration of BAY-876. Next, we examined the mRNA and protein expression of $S L C 2 A 1$ across TNBC cell lines. The response to BAY-876 is not correlated with SLC2A1 mRNA levels (Supplementary Fig. 3a) or protein level (Supplementary Fig. 3b). We then performed a systematic global profiling of the published transcriptome data for our panel of TNBC cell lines ${ }^{34}$. We profiled differentially expressed genes between BAY-876 responders and non-responders (Fig. 3a) and subjected these genes to gene set enrichment analysis (GSEA). Gene sets associated with the OXPHOS pathway stood out in the analysis as enriched in BAY876 resistant versus sensitive TNBC cell lines (Fig. 3b and Supplementary Fig. 3c), suggesting that the elevated functional mitochondrial output of resistant cells as shown in Fig. 2 is due to the increased OXPHOS gene expression signature.

GSEA analysis also revealed that the most significantly enriched pathway in resistant versus sensitive cell lines is the E2F Targets pathway (Fig. $3 \mathrm{~b}$ and Supplementary Fig. 3d), suggesting that elevated expression of genes involved in the E2F Targets pathway also correlates with resistance to GLUT1 inhibition. To further confirm this association in patient samples, we took advantage of the transcriptome data of patient TNBC tumors from TCGA data cohorts. Based on our observation of strong correlation between elevated OXPHOS metabolism and resistance to GLUT1 inhibition in TNBC cell lines, we used increased gene expression patterns of OXPHOS to discriminate BAY-876 putative-resistant samples versus putative-sensitive samples in the TCGA cohorts. Calculating the Pearson correlation coefficients between the OXPHOS pathway with other gene expression patterns revealed a strong clustering with the E2F Targets pathway, suggesting that high expression of genes involved in the E2F Targets pathway correlates with expression of OXPHOS-related genes in primary TNBC tumor samples (Fig. 3c and Supplementary Fig. 3e).

We next sought to identify a protein signature associated with the above gene expression and metabolic differences and that could predict the relative responsiveness of TNBC cells to BAY876. Using proteomics datasets from University of Texas MD Anderson Cancer Center for 12 of our TNBC cell lines ${ }^{35}$, we identified differential protein levels for a total of eight proteins, which showed a significant correlation with BAY-876 response (Fig. 3d). Most proteins (3 out of 5) enriched in resistant cell lines are components of the E2F Targets pathway, namely cyclindependent kinase inhibitor 2A (CDKN2A), cyclin E1 (CCNE1), and checkpoint kinase 2 (CHK2). Among the three proteins whose levels were increased in BAY-876-sensitive lines, the top hit was retinoblastoma tumor suppressor (RB1) protein that functions primarily as an upstream transcription factor attenuating expression levels of known E2F targets ${ }^{36,37}$. We further confirmed the association of RB1 protein levels with BAY-876 sensitivity in an independent proteomics dataset from the Princess Margaret Cancer Center ${ }^{34}$ (Fig. 3e, Supplementary Fig. 4a). We also assessed RB1 protein levels across our 17 TNBC cell lines by immunoblotting (Fig. 3f). This further confirmed the significant correlation $\left(R^{2}=0.73, p<0.0001\right)$ between RB1 protein levels and BAY-876 sensitivity (Fig. $3 g$ ). These results suggest that elevated RB1 protein levels underlie sensitivity to BAY-876 treatment, while low RB1 protein levels are associated with resistance to BAY-876 in TNBC. To test this concept, we overexpressed RB1 in two BAY-876-resistant cell lines: MDA-MB-436 and MDA-MB468 (Fig. $3 \mathrm{~h}$ and Supplementary Fig. 4b). In both cell lines, RB1 overexpression caused an increase in the ECAR/OCR ratio and markedly sensitized cells to BAY-876 treatment (Fig. 3I, j and Supplementary Fig. 4b). Conversely, RB1 knockdown in BAY876-sensitive TNBC cell lines induced higher OCR/ECAR ratios and rendered the cells refractory to the anti-proliferative effects of BAY-876 (Fig. 3k-m and Supplementary Fig. 4c-f). To examine the potential mechanism underlying RB1-dependent metabolic functions, we assessed OXPHOS gene expression in HCC1806 cells with or without RB1 knockdown. We observed that shRNA mediated silencing of RB1 induced the expression of mitochondrial genes involved in OXPHOS by quantitative reverse transcription with PCR (RT-PCR) (Supplementary Fig. 4g). This is consistent with the recent reports that RB1 loss in breast cancer induced mitochondrial protein translation and increased $\mathrm{OXPHOS}^{38}$. Collectively, our results suggest that RB1 protein levels can serve as a biomarker of response to BAY-876 treatment in TNBC cell lines.

Pharmacological inhibition of GLUT1 impedes TNBC growth. To better address the clinical relevance of this hypothesis, we tested the correlation of RB1 protein level and BAY-876 sensitivity across a panel of TNBC patient-derived samples. PDXderived organoids (PDXDOs), which are thought to better recapitulate features of breast histology and epithelial heterogeneity, and therefore serve as better tools to assess drug responses for cancer therapy (Supplementary Table 2). Thus, PDXDOs from three different TNBC patients were cultured, of which two cases are RB1-low, and one expressed RB1 based on immunoblots (Fig. 3n). In agreement with our observations in TNBC cell lines, higher RB1 expression was predictive of sensitivity to BAY-876 in PDXDOs (Fig. 3o). Some PDXDOs (PDXDO-B64 and PDXDO1963) with low-RB1 levels showed a weakened partial response to BAY-876 treatment. We postulated that this could be due to heterogeneity in RB1 expression across sub-populations of cells found in each patient-derived tumor culture. In agreement, immunostaining for RB1 protein indicates that although most of the cells are RB1 negative in PDXDO-B64 (65.7\% cells are RB1 negative), some cells showed a strong RB1 staining signal confirming a mixed population with differential RB1-related response to BAY-876 (Supplementary Fig. 4i).

We further tested the efficacy of GLUT1 inhibition and its association with RB1 protein expression in PDX-derived tumor ex vivo explant models (PDXDEs) (Fig. 4a). Unlike the organoid 
a

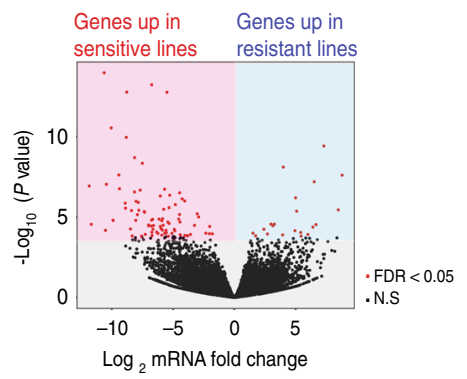

b

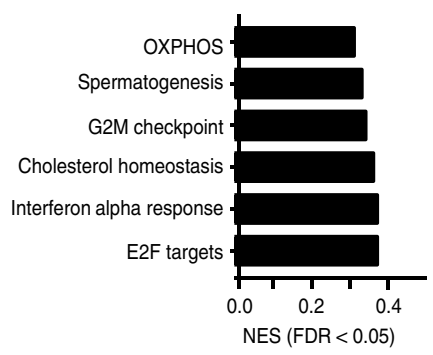

C

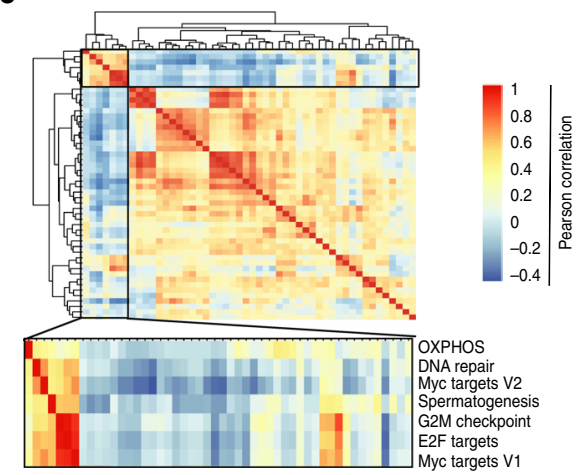

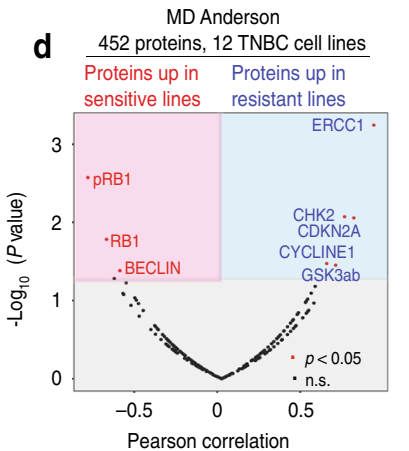

PMCC

e $\frac{218 \text { proteins, } 17 \text { TNBC cell lines }}{\text { Proteins up in Proteins up in }}$ $\begin{array}{ll}\text { Proteins up in } & \text { Proteins up in } \\ \text { sensitive lines } & \text { resistant lines }\end{array}$

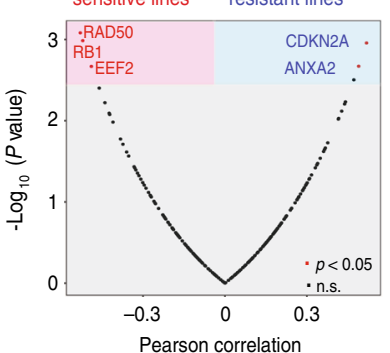

f

$\mathbf{g}$
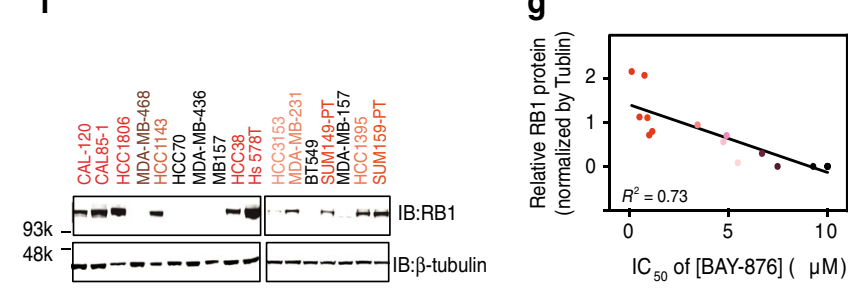
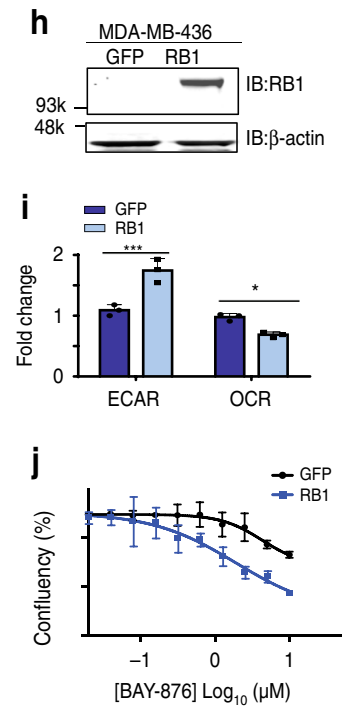
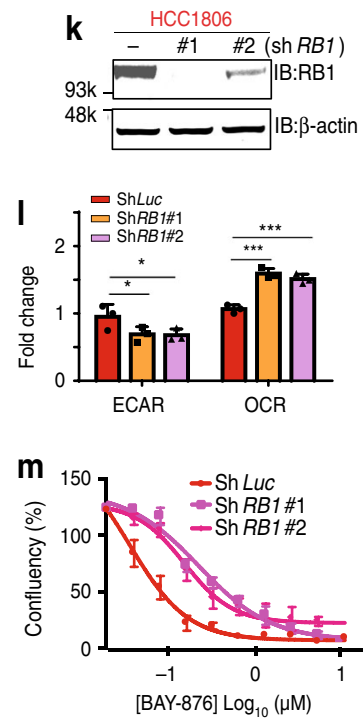
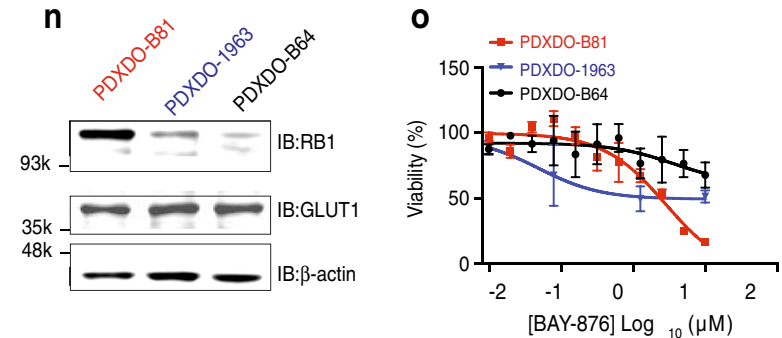

Fig. 3 RB1 protein level discriminates response to GLUT1 inhibition. a Volcano plot of $\log _{2}$ fold change for all genes significantly upregulated (red; left) in sensitive lines or in resistant lines (blue; right). b Top enriched pathways in BAY-876-resistant lines compared to BAY-876-sensitive lines based on GSEA. c Heatmap of pathways correlated with OXPHOS revealed by GSEA of TCGA RNA-sequencing data. Clusters significantly related to OXPHOS are zoomed at the bottom. Pearson's correlation coefficients between $\log _{2}$ normalized protein expression data and response of BAY-876 showing significantly associated proteins with sensitive lines (red; left) or resistant lines (blue; right) based on the dataset from $\mathbf{d}$ MD-Anderson Cancer Center and e Princess Margaret Cancer Center (PMCC). f Representative western blot showing the variable RB1 expression levels in 17 TNBC lines. $\beta$-tubulin as a loading control. $\mathbf{g}$ Correlation of RB1 protein levels and the IC 50 of BAY-876. $\mathbf{h}$ Representative immunoblot showing MDA-MB-436 cells expressing RB1 or GFP control proteins. $\beta$-tubulin as a loading control. i ECAR and OCR values were measured for MDA-MB-436 cells expressing RB1 or GFP control. $n=4$; mean \pm s.d. Two-sided Student's $t$ test. ${ }^{\star} p<0.05 ;{ }^{\star \star \star} p<0.001$. j Growth curves of MDA-MB-436 cells expressing RB1 or GFP control in the presence of indicated concentrations BAY-876 treatment for 5 days. $n=4$; mean \pm s.d. $\mathbf{k}$ Representative western blot showing HCC1806 cells transfected with shRB1 and shLUC. $\beta$-actin as a loading control. I ECAR and OCR values were measured for HCC1806 cells transfected with shRB1 and shLUC. $n=4$; mean \pm s.d. Two-way ANOVA. ${ }^{\star} p<0.05 ;{ }^{* \star \star} p<0.001$. $\mathbf{m}$ Growth curves of HCC1806 cells with control knockdown or RB1 knockdown in the presence of indicated concentrations BAY-876 treatment for 5 days. $n=4$; mean \pm s.d. $\mathbf{n}$ Western blot showing the variable RB1 expression levels in a panel of three TNBC patient-derived organoids. $\beta$-actin as loading control. $\mathbf{o}$ Cell viability assays of patient-derived organoids with indicated concentrations of BAY-876. $n=3$; mean \pm s.d. Source data are provided as a Source Data file. 
a

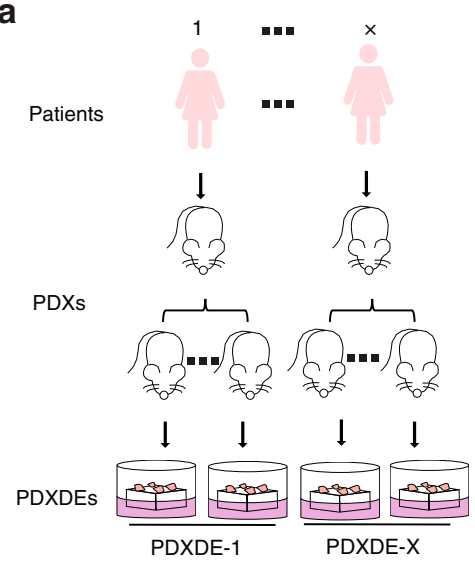

e

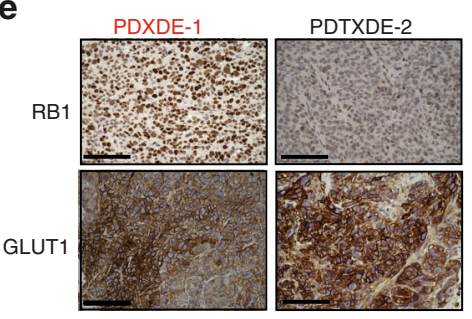

f

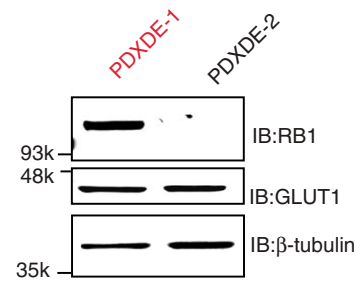

i $\quad$ PDXDE-1 (RB1 high)

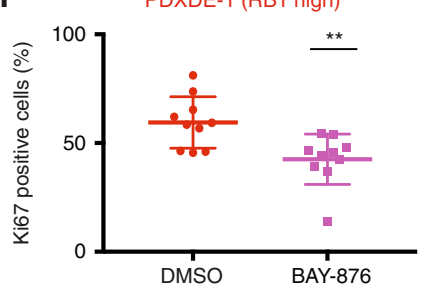

j $\quad$ PDXDE-1 (RB1 high)

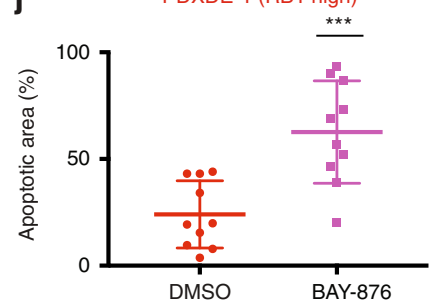

b

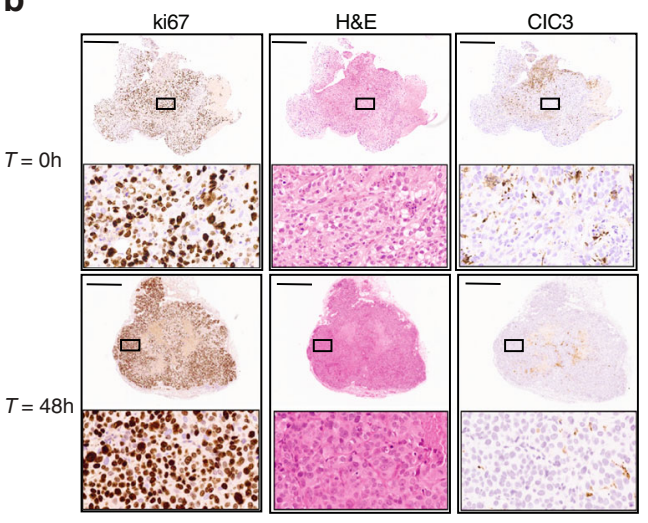

c
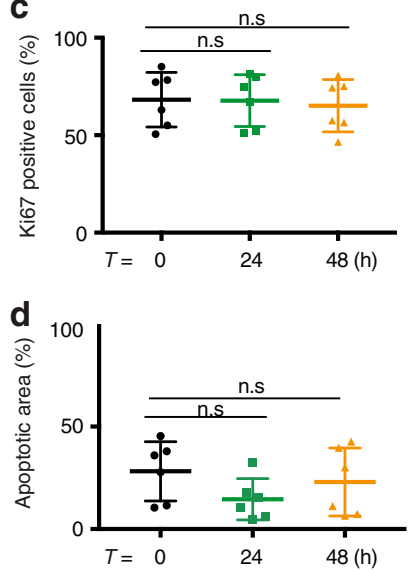

g

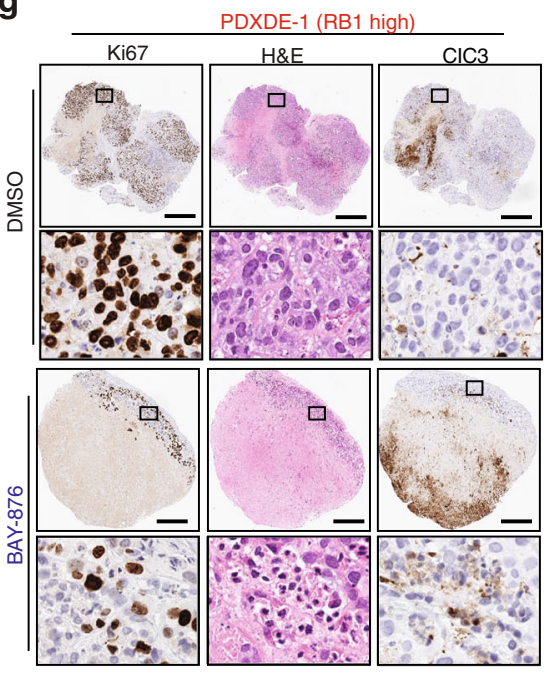

h

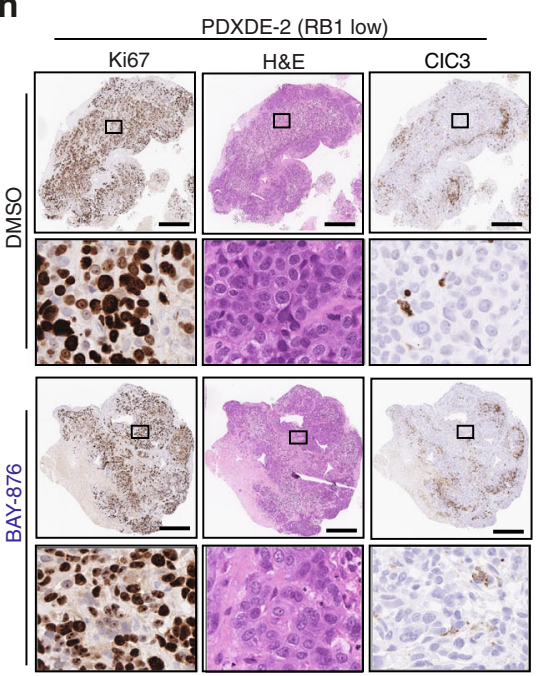

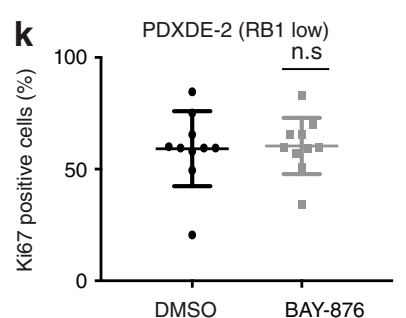

PDXDE-2 (RB1 low)

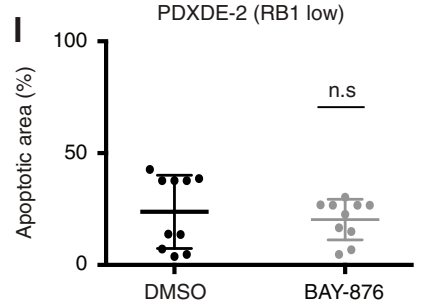

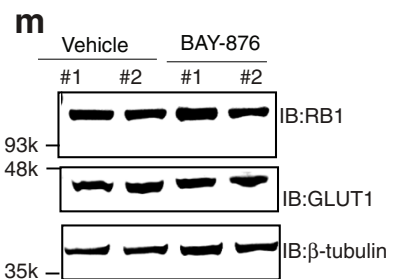
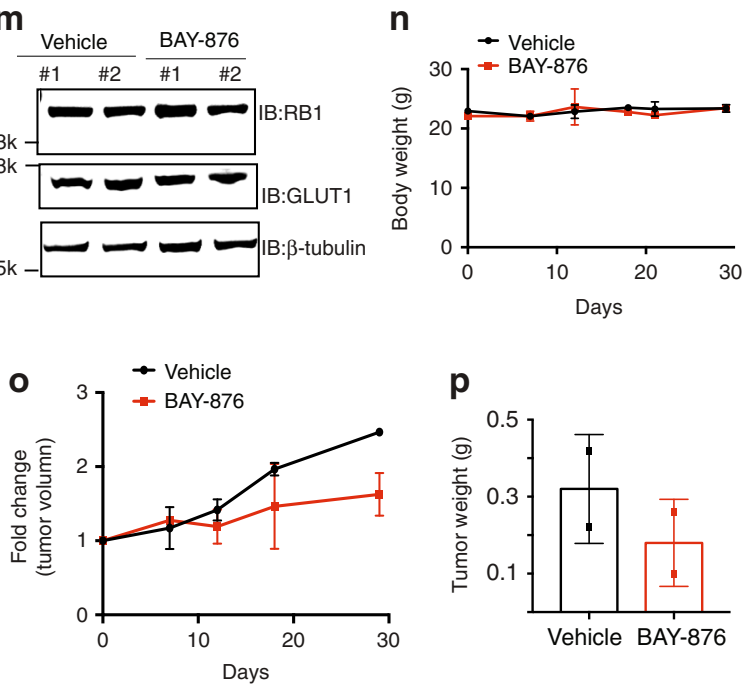

p

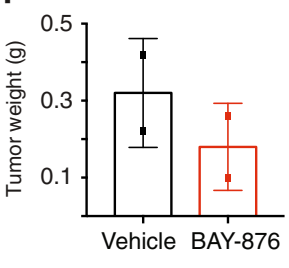

cultures generated from tumors that are minced and enzymatically digested to dissociate cells, PDXDEs are cultured in their native format (intact tissue) and therefore preserve their original patient-specific 3D architecture and tumor microenvironment (Supplementary Table 2). In this way, these models are more analogous to PDX models while avoiding the significant associated costs and timeframes. The PDXDE format has additional advantages over cell lines, organoid and PDX models, such as its high take rate regardless of the aggressiveness of tumor stage, and its ability to culture tumor samples within a physiological 3D architecture (as detailed in Supplementary Table 2). Therefore, PDXDEs have been shown to accurately predict patient-specific responses to multiple drugs and to mimic in vivo results in breast and other cancers. An important advantage of PDXDE models is that they can be used to assess compounds with sub-optimal pharmacological properties (such 
Fig. 4 RB1 levels dictates BAY-876 sensitivity in patient samples. a Schematic of pre-clinical PDXDE trial. PDXDEs established from TNBC patients were evaluated for response to BAY-876 treatment. b Representative images of explants during ex vivo culture time range assessed by ki67 staining, H\&E staining, and cleaved caspase 3 (CIC3) staining. Scale bars represent $500 \mu \mathrm{m}$. Indicated area is zoomed in $5 \times$ at the bottom. Ex vivo culture for $48 \mathrm{~h}$ did not significantly change the $\mathbf{c}$ cell proliferation of explants assessed by Ki-67 staining; and $\mathbf{d}$ the apoptosis of explants. $n=6$; mean \pm s.d. e Representative IHC staining images of GLUT1 and RB1 for PDXDE-1, PDXDE-2. f Representative immunoblotting showing RB1 and GLUT1 expression levels in PDXDE-1 and PDXDE-2. g Representative IHC staining images including ki67 staining, H\&E staining, and CIC3 staining of RB1-positive PDXDE-1. Scale bars represent $500 \mu \mathrm{m}$. Indicated area is zoomed in 10x at the bottom. h Representative IHC staining images for PDXDE-2. Scale bars represent $500 \mu \mathrm{m}$. Indicated area is zoomed in $10 \times$ at the bottom. i BAY-876 treatment resulted in regression of PDXDE-1 growth assessed by ki67 staining. $n=10 ;$ mean \pm s.d. Two-sided Student's $t$ test. ${ }^{* \star *} p<0.0001$. j BAY-876 treatment resulted in increased apoptosis of PDXDE- 1 assessed by CIC3 staining. $n=10 ;$ mean \pm s.d. Two-sided Student's $t$ test. ${ }^{\star \star \star} p<0.001$. k BAY-876 treatment did not result in significant change of PDXDE-2 growth assessed by ki67 staining. $n=11 ;$ mean \pm s.d. Two-sided Student's $t$ test. I BAY-876 treatment did not lead to significant changes of apoptosis of PDXDE-2 assessed by $\mathrm{CIC} 3$ staining. $n=10 ;$ mean \pm s.d. Two-sided Student's $t$ test. $\mathbf{m}$ Representative immunoblotting showing RB1 and GLUT1 expression levels in PDX. Data shown were $\mathbf{n}$ body weights over the course of experiment; $\mathbf{o}$ growth curves of tumor volumes and $\mathbf{p}$ tumor weight of end point xenografts. Source data are provided as a Source Data file.

as pharmacokinetic (PK)/pharmacodynamic (PD) and safety profiles) that may limit the maximal dose or exposure that can be safely achieved in vivo mouse studies, such as the chemical probe BAY-876.

We first established the appropriate culture conditions to maintain TNBC PDXDEs. As illustrated by hematoxylin and eosin (H\&E) staining, tissue architecture and morphology of PDXDEs cultured for up to $48 \mathrm{~h}$ on gelatin sponges were consistent with the original $(T=0 \mathrm{~h})$ tumor tissue (Fig. $4 \mathrm{~b}$ and Supplementary Fig. 5). Tumor cells are present in the surrounding stroma, demonstrating maintenance of the PDX architecture (Supplementary Fig. 5). The proliferative capacity of explants was assessed using the immunohistochemistry marker ki67, with a change of $25 \%$ or more considered to be a significant response ${ }^{39}$. No significant change in the number of ki67-positive cell nuclei was observed between $T=0 \mathrm{~h}$ and $48 \mathrm{~h}$ of culture $(T=48 \mathrm{~h})$ for matched tissues (Fig. 4c). Furthermore, as shown by immunohistochemistry staining for the apoptotic marker cleaved caspase3 (CIC3), no significant differences in the proportion of apoptotic cells was observed in PDXDEs cultured over a $48 \mathrm{~h}$ period (Fig. 4d). Altogether, our results demonstrate that PDXDEs are viable over the experimental period of $48 \mathrm{~h}$.

Next, we quantified the RB1 protein level by RB1 immunohistochemistry staining in PDXDEs from six different patients (Supplementary Fig. 6a ${ }^{40,41}$. Each PDXDE case was assigned a score of $0(<10 \%$ of cells positively stained), 1 (if $>10 \%$ and $<50 \%$ of cells positively stained), or 2 ( $>50 \%$ of cells positively stained), representing RB1-negative, intermediate, or positive PDXDEs, respectively (Supplementary Fig. 6a). The RB1-positive (PDXDE-1) and RB1-negative explants (PDXDE-2) were then used to evaluate the efficacy of GLUT1 inhibition. Each of these PDXDEs expressed GLUT1 at similar levels (Supplementary Fig. 6b). RB1 and GLUT1 expression levels in both PDXDEs were confirmed by immunoblotting (Fig. 4e, f). Consistent with our cell line and PDXDO results, BAY-876 $(3 \mu \mathrm{M})$ treatment abrogated the proliferation in 70\% (7/10) PDXDE-1 RB1-positive explants within $48 \mathrm{~h}$ as measured by ki67 staining when compared to vehicle-treated PDXDEs (Fig. 4g, Supplementary Fig. 7). Although intertumoral heterogeneity to BAY-876 response was observed across these explants, PDXDE-1 explants showed a significant overall reduction in ki-67 proliferation $(p<0.01)$ upon BAY-876 treatment (Fig. 4i). Moreover, cleaved caspase-3 staining revealed a significant increase in apoptosis in BAY-876-treated PDXDE-1 (Fig. 4j and Supplementary Fig. 7). Conversely, no significant changes in cell proliferation or apoptotic markers were observed following BAY876 treatment in the RB1-negative PDXDE-2 explants (Figs. 4h, k, 1 and Supplementary Fig. 8).

Finally, we evaluated the anti-tumor efficacy of BAY-876 using a PDX model derived from a TNBC patient that expresses both RB1 and GLUT1 (Fig. 4m). Animals with tumors of $100 \mathrm{~mm}^{3}$ were randomized into two groups, which were orally treated with vehicle or BAY-876, respectively. Once-daily treatment with 2 mg-kg per day of BAY-876 for 30 days impaired tumor growth with no significant loss of body weight (Fig. $4 \mathrm{n}, \mathrm{o})^{14}$. Tumor weights after 30 days of treatment were significantly less in mice treated with BAY-876 compared to vehicle-treated mice (Fig. 4p). Collectively, our findings across a range of pre-clinical PDXderived models provide a strong rationale for selecting GLUT1 inhibition as a therapeutic strategy on the basis of RB1 protein expression level against TNBC, in support of further evaluation of strategies to inhibit GLUT1 in TNBC patients.

\section{Discussion}

In this study, we identify a dependency of a subset of TNBCs on GLUT1 function, and relate this dependency to the distinct basal cellular bioenergetic profile. Our findings suggest a mechanistic basis by which cells with higher glycolysis/OXPHOS rate are susceptible to GLUT1 inhibition. These results reveal important information to consider for the design of therapeutic strategies for TNBC, given the known heterogeneity within TNBCs, both genetically and metabolically ${ }^{5}$. This is also supported by the recent observations of metabolic heterogeneity across diverse cancer cell lines at both the unperturbed and the perturbed states $^{19}$. The latter study suggests that heterogeneous metabolic dependencies across cancer cell lines underlie differential therapeutic vulnerabilities associated with specific cancer genotypes ${ }^{19}$. In addition, direct targeting of metabolic states in TNBC has shown promising results. For example, inhibitors that target glutathione biosynthesis, folate receptor, fatty acid oxidation as well as glutamine metabolism were shown to suppress tumor growth in TNBC ${ }^{42-45}$. However, successful clinical translation of these results is hampered by issues such as metabolic plasticity ${ }^{18}$.

Metabolic plasticity need not be inherent but may be adaptive, based on the stage of tumor progression, tumor microenvironment, such as nutrient availability, and the type of treatment administered ${ }^{46,47}$. It is likely that in many cases in which glycolysis is inhibited, cells will respond by increasing other alternative metabolic pathways 48,49 . Supporting this, our results demonstrated that GLUT1 inhibition triggered metabolic reprogramming toward OXPHOS in BAY-876-resistant TNBC cell lines. This metabolic adaptation in conjunction with the use of alternative nutrients, such as glutamine overcomes the inhibition of glucose metabolism in our BAY-876-resistant TNBC cell lines. Thus, glutamine deprivation sensitizes the resistant cells to BAY876 inhibition. This synergism between glycolysis inhibitors combined with OXPHOS inhibition suggests a strong rationale for using two or more drugs targeting different metabolic pathways to achieve superior therapeutic benefits for TNBC treatment ${ }^{50}$. 
Many relationships between cancer metabolism and genotypes have been established in which genomic alterations are used to identify metabolic differences ${ }^{20,21}$. Mutations that activate oncogenes or inactivate tumor suppressors can significantly affect activities of metabolic enzymes and have a key role in aerobic glycolysis of cancer ${ }^{20,21}$. Phosphatidylinositol $3^{\prime}$-kinase (PI3K) ${ }^{51}$, phosphatase and tensin homolog (PTEN) ${ }^{52}, \mathrm{Myc}^{53}$ and $\mathrm{p} 53^{54} \mathrm{can}$ all impact cellular glucose metabolism. Here, we discovered the significant correlation between RB1-E2F Targets pathway and OXPHOS in both cell lines and TNBC primary tumors. TNBC cells that lack RB1 protein have increased expression of OXPHOS genes and accordingly increase mitochondrial respiration, ultimately leading to resistance to GLUT1 inhibition. This metabolic role of RB1 in TNBC is underappreciated, but consistent with recent reports suggesting that RB1 loss stimulates mitochondrial function rather than anaerobic metabolism by activating E2F $\operatorname{targets}^{38,55}$. These connections are especially interesting given the rapidly growing evidence that $\mathrm{RB} 1$ is needed for cells to maintain a normal metabolic balance, and that the loss of RB1 leads to reprogramming of specific pathways ${ }^{56-58}$. Metabolic adaptations are thought to enhance the ability of cancer cells to sustain the metabolic intermediates for cell survival in multidrug-resistant states, providing a rationale for the potential use of metabolic inhibitors, such as BAY-876, for the post-chemotherapy treatment in drug-resistant $\mathrm{TNBC}^{59,60}$. Our results outline a clinically feasible scenario to use RB1 protein level as a biomarker to stratify the TNBC patients for targeted therapy.

Bioinformatic analyses of clinical samples illustrate the relevance of our findings to patients with breast cancer. Analysis of breast cancer patients revealed heterogeneous RB1 expression at both mRNA (Supplementary Fig. 9a, b) and protein levels (Supplementary Fig. 9c) in basal tumors. Approximately $47 \%$ of basal breast tumors are RB1 positive based on higher than average levels of protein expression (Supplementary Fig. 9c), suggesting that this sub-population of TNBC patients could benefit from GLUT1 inhibition. However, 20-30\% of these basal-like tumors are RB1 low ${ }^{23,55,61}$ (Supplementary Fig. 9a-c). Within this RB1low subtype, RB1 loss stimulates mitochondrial biogenesis with higher OXPHOS rates ${ }^{38,55}$. Consistent with our preclinical results, RB1 expression inversely correlates with higher expression of OXPHOS genes in individual TNBC patient tumors (Supplementary Fig. 9d). Together, these data suggest that drugs targeting mitochondrial function, may also exert anti-tumor effects in RB1-low TNBC patients ${ }^{38,55}$. Thus, our results highlight an additional role for RB1 tumor suppressor protein, in addition to the regulation of cell proliferation and cell cycle, as a key regulator of cell metabolism and determinant of TNBC response to the metabolic inhibitor BAY-876.

In summary, we have discovered an RB1 protein-dependent metabolic addiction to GLUT1 function in a subset of TNBCs, identifying BAY-876 as an effective agent to block growth in patient-derived models that express RB1 protein. Our findings also suggest the importance of considering the heterogeneity of RB1 protein levels in the development of personalized metabolic chemotherapeutic approaches toward TNBC treatment.

\footnotetext{
Methods

Cell culture. Human breast cancer cell lines were obtained from American Type Culture Collection (ATCC, Teddington, UK). All cells were routinely cultured in RPMI 1640 (Life Technologies 11965), or Dulbecco's modified Eagle's medium (Gibco) supplemented with 10\% FBS recommended by suppliers. The cell lines were authenticated by short-tandem-repeat (STR) analysis and matched to the German Collection of Microorganisms and Cell Cultures (DSMZ) database, and they were used for no more than 25 passages after STR typing. Mycoplasma tests were routinely performed using MycoAlert Mycoplasma Detection Kit (Lonza, Basel, Switzerland).
}

GLUT1 inhibitors. GLUT1 inhibitors including STF31 (millipore sigma \#SML1108), WZB-117 (millipore sigma \#SML0621), and Fasentin (millipore sigma \#F5557) were purchased from Millipore Sigma. BAY-876 was provided by BAYER via the SGC (https://www.thesgc.org/chemical-probes) and also purchased from MedChemExpress (Cat. no. HY-100017).

SLC2A1 mRNA expression analysis. TCGA mRNA expression was downloaded from the Xena browser ${ }^{62}$. METABRIC mRNA expression was downloaded using the MetaGx R package. Genefu R package ${ }^{63}$ was used to classify all samples into PAM50 molecular subtypes. The Wilcoxon rank sum test was used to measure the significance of difference between TNBC vs. non-TNBC samples and also between the different specific subtypes.

TCGA data analysis. Breast adenocarcinoma TCGA data was retrieved from Firehose using the GSVA R package ${ }^{64}$. We identified enrichment of Hallmark gene sets $^{65}$ for each TNBC tumor sample in TCGA using their RNAseq profiles. The enrichment was conducted using the ssGESA method as part of GSVA R pack$\mathrm{age}^{64}$. The correlation between the Hallmarks were then calculated using Spearman's rank correlation. Rows and columns were hierarchically clustered by Euclidean distance with complete linkage.

Proliferation and colony formation assays. TNBC control and gene knocked down cells (500 cells per well) were seeded in a 384-well plate and transferred to Incucyte ZOOM analysis system (Sartorius) that was maintained at $37^{\circ} \mathrm{C}$. Growth profile was monitored by $\times 10$ objective every $6 \mathrm{~h}$ using Incucyte software 2016A with an integrated confluence algorithm until $72 \mathrm{~h}$. Standard mode per well was used to collect images in phase-contrast mode and averaged to provide a representative statistical measure of the well confluency. For colony-formation assays, 500-1000 cells were seeded in six-well plates. At the indicated time point (usually 10-14 days), cells were fixed with $80 \%$ methanol and stained with crystal violet solution overnight. All experiments were performed in triplicate.

NHA cell assay. NHA or normal human astrocytes (Lonza, catalog number CC2565) were plated in 384-well CELLBIND plates (Corning) at 750 cells per well. Astrocyte basal media was supplemented with rhEGF, insulin, ascorbic acid, Lglutamine, and FBS as per the manufacturer's protocol. After $24 \mathrm{~h}, \mathrm{BAY}-876$ was added to the plate at a final concentration of $5 \mu \mathrm{M}$. Live cell imaging data was collected over a 14-day period using the Incucyte ZOOM Live-Cell Analysis System (Essen Biosciences). The culture media was refreshed every 5 days with $1 \times$ chemical probe concentration. To determine NHA response to probe addition, the average confluency of triplicates for each probe was normalized to the average confluency of vehicle (DMSO)-treated cells.

Small interfering RNA (siRNA)-mediated gene knockdown. siGENOME siRNA targeting SLC2A1 (L-007509-02) and a non-targeting siRNA pool (D-001206-14) control, were purchased from Dharmacon (Thermo Scientific, Hemel Hempstead, UK). Cells were transfected with $25 \mathrm{nmol} / \mathrm{L}$ siRNA using Lipofectamine RNAiMax transfection reagent (Invitrogen, Life Technologies, Paisley, UK), following the manufacturer's instructions.

Western blot analysis. Cells were lysed directly in $1 \times$ lysis buffer $(50 \mathrm{mmol} / \mathrm{L}$ Tris-HCl pH 6.8, 2\% SDS, $10 \%$ glycerol, $2.5 \% \beta$-mercaptoethanol, and $0.1 \%$ bromophenol blue). Alternatively, cells were lysed by scraping them into a $\mathrm{pH} 7.4$ lysis buffer containing $1 \% \mathrm{NP}-40$ (Sigma-Aldrich, Gillingham, UK), $50 \mathrm{mmol} / \mathrm{L}$ Tris, $10 \%$ glycerol, $0.02 \% \mathrm{NaN}_{3}, 150 \mathrm{mmol} / \mathrm{L} \mathrm{NaCl}$, and a cocktail of phosphatase and protease inhibitors (Sigma-Aldrich, Gillingham, UK). Snap-frozen tumor tissues were suspended in a $\mathrm{pH} 7.4$ lysis buffer containing $50 \mathrm{mmol} / \mathrm{L}$ Tris base, 150 $\mathrm{mmol} / \mathrm{L} \mathrm{NaCl}, 2 \%$ TritonX-100, $1 \%$ SDS, $10 \mathrm{mmol} / \mathrm{L}$ EDTA, and a cocktail of phosphatase and protease inhibitors (Sigma-Aldrich, Gillingham, UK). Tissue destruction was done with the bullet blender homogenizer (Next Advance, New York, USA). $20-100 \mu \mathrm{g}$ of proteins were separated in reducing conditions $(2.5 \% \beta$ mercaptoethanol) by SDS-PAGE (SDS-polyacrylamide gel electrophoresis) and transferred to nitrocellulose membranes (Bio-Rad, Hemel Hempstead, UK) for further processing, following standard western blotting procedures.

Primary antibodies used in this study were: anti-GLUT1 rabbit monoclonal (EPR3915) (ab115730) (1:1000), anti-RB1 rabbit monoclonal (EPR) (ab181616) (1:2000), anti-beta actin (ab16039) (1:5000). The secondary antibodies are goatanti-rabbit (IR800 conjugated, LiCor no. 926-32211) and donkey anti-mouse (IR 680, LiCor no. 926-68072) antibodies (1:5000). Odyssey Licor system were used to scan membranes and ImageJ was used to quantify western blotting results by densitometry

Cell cycle assay. Exponentially growing cells in six-well plates were treated with $3 \mu \mathrm{M}$ BAY-876 or DMSO for $24 \mathrm{~h}$ before cell cycle analysis using allophycocyanin (APC) BrdU Flow kit (BD Pharmingen). Briefly, cells were incubated with $10 \mu \mathrm{M}$ BrdU for $6 \mathrm{~h}$ before fixation, permeabilization, and staining with APClabeled antiBrdU antibody and 7-aminoactinomycin D (7-AAD) according to the manufacturer's instructions. Cells were then analyzed using a BD FACScan flow 
cytometer and the percentage of live cells in each cell cycle stage was determined using FlowJo software (version 9.3.1). Experiments were performed in duplicate and the percentage of cells in each stage was compared between the treated and untreated samples for each cell line using a two-tailed $t$ test. $p$ values $\leq 0.05$ were considered significant.

Cell apoptosis assay. For cell death assays TNBC cells ( 3000 cells per well) were seeded in 96-well plates and incubated for $24 \mathrm{~h}$ at $37^{\circ} \mathrm{C}$. The cells were then treated either with DMSO or BAY-876 for 5 days. Post treatment, Caspase-3/7 green apoptosis assay reagent (Sartorius \#4440) was added to the cells and transferred to Incucyte ${ }^{\circledR}$ ZOOM 2 FLR system and analyzed using 2016 integrated software.

Mitochondrial respiration and glycolysis rate measurements. Bio-energetic studies (OCR: oxygen consumption rate and ECAR) were measured using a Seahorse XFe96 Extracellular Flux Bioanalyzer (Agilent). TNBC cells $\left(10^{4}\right)$ were seeded and cultured for $24 \mathrm{~h}$. The medium was then replaced with DMEM $(25 \mathrm{mM}$ glucose, $2 \mathrm{mM}$ glutamine, no sodium bicarbonate) $\mathrm{pH} \sim 7.4$ and incubated for $1 \mathrm{~h}$ at $37^{\circ} \mathrm{C}$ in a $\mathrm{CO}_{2}$-free incubator. For the mitochondrial stress test (Seahorse 101706100 ), oligomycin, trifluoromethoxy carbonylcyanide phenylhydrazone (FCCP), and a mixture of antimycin and rotenone were injected to final concentrations of 2, 0.5, and $4 \mu \mathrm{M}$, respectively. For the glycolysis stress test (Seahorse 102194-100), glucose, oligomycin and 2-deoxyglucose were injected to final concentrations of 10 $\mathrm{mM}, 2 \mu \mathrm{M}$ and $100 \mathrm{mM}$, respectively. OCR and ECAR were normalized to cell number as determined by CyQUANT NF Cell proliferation assay kit (ThermoFisher Scientific, C7026). OCR and ECAR values were used to compute basal respiration, spare capacity, proton leak, ATP production, glycolysis, and glycolytic capacity. Calculations from four independent experiments were performed using ExcelMacro Report Generator Version 3.0.3 provided by Seahorse Biosciences and two-sided Student's $t$-test computed using GraphPad.

Measurement of total cellular ROS. A dichlorofluorescin diacetate (DCFDA) cellular ROS detection assay (abcam, ab113851) was used to measure total ROS activity within the cells. A total of $2.5 \times 10^{4}$ cells per well were seeded in a 96-well plate and allowed to attach overnight. The cells were then stained with $25 \mu \mathrm{M}$ DCFDA for $45 \mathrm{~min}$ at $37^{\circ} \mathrm{C}$. After staining, the cells were washed and measured using a microplate reader with fluorescence $(\mathrm{Ex} / \mathrm{Em}=485 / 535 \mathrm{~nm})$.

Metabolite measurements. Glucose, lactate, and glutamine were quantified simultaneously using Bioprofile Flex analyzer (Nova Biomedical). Briefly, $300 \mu \mathrm{L}$ of the cell/debris free culture medium was used to measure glucose/glutamine consumption and lactate production. The values were normalized to the cell number.

Gene set enrichment analysis. Transcriptome data available for our tested 17 cell lines were processed using the Kallisto pipeline ${ }^{34}$. Drug activity was extracted from growth curves produced by IncuCyte assay. IncucyteDRC R package was used to process the growth curves and obtain concentration and viability normalized to control near confluence point ${ }^{66}$ and PharmacoGx R package was then used to obtain drug activity measures such as $\mathrm{IC}_{50}$ and area-above-the-dose-response curve (AAC) ${ }^{67}$. Genes were ranked based on the Pearson correlation coefficients between the measured drug activity $\left(\mathrm{IC}_{50}\right)$ and individual gene expression levels over all 17 cell lines. Hallmarks gene sets were downloaded from MsigDB ${ }^{65}$ and the piano R package was used to produce the GSEA results. Pathways enrichment plots were generated using fgsea $R$ package ${ }^{68}$.

Proteomics analysis. MD Anderson protein expression data was downloaded from ref. ${ }^{35}$. Princess Margaret Cancer Center protein expression data was downloaded from ref. ${ }^{34}$. Pearson correlation coefficients were calculated to measure the degree of association between the measured drug activity $\left(\mathrm{IC}_{50}\right)$ and individual protein expression levels over all samples.

Inducible ectopic expression of RB1. eGFP as control or RB1 were cloned into the $\mathrm{Rc} / \mathrm{CMV}$ vector (Addgene plasmid 1763). The entire transfection reaction was transfected into 2-3 million cells through Nucleofection (SF Solution EN120-4D Nucleofector, Lonza; http://www.lonzabio.com/cell-biology/transfection/). The transfection efficiency of this procedure resulted in the transient expression of the transfected gene lasting up to $10-12$ days in $60-80 \%$ of TNBC cells.

Lentiviral mRNA targets. Two independent shRNA vectors targeting RB1 were obtained from Addgene (Addgene ID: 25640 and 25641). Lentivirus was produced using standard virus production methods by co-transfecting target and packaging plasmids into HEK293T cells. Cell lines were then transduced with $0.45 \mu \mathrm{M}$ filtered and ultracentrifuge-concentrated viral particles with polybrene $\left(8 \mu \mathrm{g} \mathrm{ml}^{-1}\right)$. After $16 \mathrm{~h}$ of transduction, the media was changed for fresh regular growth media, and $48 \mathrm{~h}$ later selection started using puromycin $\left(0.2-0.6 \mu \mathrm{g} \mathrm{ml}^{-1}\right)$. After selection was complete in $72 \mathrm{~h}$, cells were termed stably transduced.
Quantification of RNA expression (qRT-PCR). mRNA was extracted from cells using the RNeasy Isolation Kit (Qiagen). According to the manufacturer's protocol for single-stranded cDNA synthesis, $500 \mathrm{ng}$ of the total RNA was reverse transcribed using iScript cDNA synthesis kit (BIO-Rad). cDNA samples were amplified using SYBR green supermix (BIO-Rad), in a white 96-multiwell plate by LightCycler 96 system instrument (Roche) according to the manufacturer's protocol. To quantify the data, the comparative Ct method was used. Relative quantity was defined as $2-\Delta \Delta \mathrm{Ct}$ and $\beta 2$-microglobulin was used as a reference gene. qRT-PCR primers are available in Supplementary Table 3.

Statistics analysis. All measurements were taken from distinct samples/biological replicates. Data are presented as mean \pm SD. With the exception of gene and protein expression in clinical cohorts, the statistical analyses were performed using Graphpad Prism 6 with at least three biological replicates. For TCGA and METABRIC analyses (Fig. 1a-c, Supplementary Fig. 1d, Supplementary Fig 9a-c), Wilcoxon rank sum test was used to measure the significance of difference between TNBC vs. non-TNBC samples and also between the different specific subtypes. Unless otherwise indicated in the figure legends, differences were analyzed by unpaired two-tailed $t$ test between two groups (Figs. $2 \mathrm{~b}$ and $4 \mathrm{i}-\mathrm{l}$ ) and by two-way ANOVA (Figs. 1f-h, j, 2c-e, g, 3i, j, Supplementary Fig. 1i, j, Supplementary Fig. 2a-f, Supplementary Fig. 4g) for differences between more than two groups. $p$ values of $<0.05$ were considered significant, and ${ }^{*} p<0.05,{ }^{* *} p<0.01$, and ${ }^{* * *} p<0.001$.

Immunohistochemistry staining. Paraffin sections at $4 \mu \mathrm{m}$ thickness were dried at $60^{\circ} \mathrm{C}$ oven for $2 \mathrm{~h}$ before staining. The immunohistochemistry (IHC) was performed according to the manufacturer's guidelines using BenchMark XT-an automated slide stainer (Ventana Medical System). Glutl (Roche \#06419178001) IHC was done with mild antigen retrieval (CC1, pH8.0, \#950-124), 32 min antibody incubation and Ventana iView DAB Detection Kit (\#760-091). The dilution for RB (BD \#554136) was 1:1600 with 64 min antigen retrieval, 32 min antibody incubation and Optiview Detection Kit (\#860-099). Antibody dilution for Ki67 (Dako M7240, clone MIB1) was 1:100 with standard antigen retrieval, 60 min antibody incubation and Ultraview Detection kit (\#760-500). Dilution for cleaved caspase 3 (CST \#9661) was 1:500 with standard antigen retrieval, 32 min antibody incubation and iView Detection Kit. The slides were counterstained with Harris hematoxylin, dehydrated in graded alcohol, cleared in xylene and coverslipped in Permount.

Image capture and quantification of immunostaining. Stained slides were subjected to whole slide imaging using an Aperio ScanScope AT2 at $\times 20$ magnification. Digital images were loaded into Definiens TissueStudio 4.3 software (Definiens Inc., Munich, Germany), and individual tissue slices were identified away from the slide background. A machine-learning classifier was trained to identify viable tumor tissue from stroma, necrosis, and artifact, with manual quality correction to reclassify any mislabeled regions. Stain separation was used to isolate hematoxylin counterstain from $\mathrm{DAB}$ antibody-specific stain, and the hematoxylin channel was subjected to computer-vision-based segmentation algorithm to identify individual nuclei, with a watershed step to break apart closely packed nuclei. Cell simulation grew a small region around each nucleus to simulate a cytoplasm, and the resulting cell objects were classified into "negative", "low" "medium', and "high' DAB intensity thresholds, based on comparison to clear positive and negative controls. Per-cell statistics were reported for each tissue region within each slide, and used to calculate the proportion of positive cells for each region of interest.

Generation and maintenance of PDXDO-1963. PDX were generated in NSG mice as above from TNBC biopsies and used under an REB-approved research protocol (UHN:15-9481). PDX tumors were excised from mice at passage 2, minced and digested in Advanced DMEM/F12 (ThermoFisher) containing $1 \mathrm{X}$ GlutaMAX, $10 \mathrm{mM}$ HEPES, $1 \mathrm{X}$ antibiotic-antimycotic and $500 \mathrm{\mu g} \mathrm{ml}^{-1}$ Liberase TH (Sigma-Aldrich) in Miltenyi MACS C tubes. Digestion was performed using the gentle MACS Octo Dissociator (Miltenyi). Digested tissue was processed and plated in reduced growth factor basement membrane extract (BME) Type 2 (Cultrex) in media ${ }^{69}$. The organoids were confirmed to be of human origin and devoid of mouse cells by flow cytometry. Organoids were passaged approximately every 3 weeks; they were dissociated to single cells with TrypLE Express (ThermoFisher) and by manual pipetting, passed at a ratio of 1:12 and re-embedded in BME. For cell viability assays, organoids were dissociated and plated at 3000 cells per well in 384-well plates pre-coated with $8 \mathrm{ml}$ BME. Cells were grown for 4 days; then treated with the indicated concentrations of BAY- 876 for 5 days. Cell viability was assessed using Cell Titer Glo 3D (Promega).

Generation and maintenance of PDXDO-B81 and PDXDO-B64. PDX tumors were excised from mice, minced and digested in Advanced DMEM/F12 (ThermoFisher) containing $1 \mathrm{X}$ GlutaMAX, $10 \mathrm{mM}$ HEPES, $1 \mathrm{X}$ antibiotic-antimycotic and $500 \mu \mathrm{g} \mathrm{ml}^{-1}$ Liberase TH (Sigma-Aldrich) in Miltenyi MACS C tubes. Digestion was performed using the gentle MACS Octo Dissociator (Miltenyi). Digested tissue was processed and plated in reduced growth factor BME Type 2 (Cultrex) in media ${ }^{69}$. The organoids were confirmed to be of human origin and devoid of mouse cells by flow cytometry. Organoids were passed approximately every 3 weeks; they 
were dissociated to single cells with TrypLE Express (ThermoFisher) and by manual pipetting, passed at a ratio of 1:12 and re-embedded in BME.

Ex vivo tissue explant preparation and culture. When the PDXs (preferably first generation PDX) reached $\sim 500 \mathrm{~mm}^{3}$, they were excised from the mice. Tumors were then cut into $2 \times 2 \times 2 \mathrm{~mm}^{3}$ tissue explants and cultured on gelatine sponges in 12-well tissue culture plates for specific time points as indicated in the text. The DMEM culture media (Gibco) containing 20\% FBS (Gibco), $1 \mathrm{mM}$ sodium pyruvate (Biological Industries), $2 \mathrm{mM}$ L-glutamine (Biological Industries), $1 \%$ penicillin/streptomycin/amphotericin (Biological Industries), $0.1 \mathrm{mM} \mathrm{MEM} \mathrm{non-}$ essential amino acids (Biological Industries), $10 \mathrm{mM}$ HEPES (Biological Industries), $1 \%$ BIO-MYC (Biological Industries) and $50 \mu \mathrm{g} \mathrm{ml}^{-1}$ gentamicin (Gibco). For drug treatment, the $2 \times 2 \times 2 \mathrm{~mm}^{3}$ explants were treated with $3 \mu \mathrm{M}$ BAY-876 for indicated time in $37^{\circ} \mathrm{C}, 5 \% \mathrm{CO}_{2}$.

Mouse PDX studies. All animal experiments were reviewed and approved by the Animal Care Committee at the University Health Network in Toronto. For in vivo dosing experiments, BAY-876 was purchased from MedChemExpress (Cat. no. HY-100017). PDX tumors were subcutaneously implanted into SCID mice following standard procedure. BAY-876 was prepared at 10\% NMP, 90\% PEG300, and administered to the mice when tumors reach $100 \mathrm{~mm}^{3}$ in volume. The treatments were performed once daily by oral gavage for a total of 30 days. Body weights and tumor growth were measured once a week over the course of treatment until the endpoint was reached.

Protein expression data analysis. TCGA Breast cancer RPPA data was downloaded from the Cancer Proteome Atlas ${ }^{70}$. Genefu R package ${ }^{63}$ was used to classify all samples into PAM50 molecular subtypes. The Wilcoxon rank sum test was used to measure the significance of difference between basal vs. non-basal samples and also between the different specific subtypes.

\section{Analysis of association between RB1 expression and OXPHOS gene expres- sion. TCGA breast cancer mRNA expression was downloaded from the Xena browser ${ }^{62}$. TCGA patients were subsetted to those showing high and low levels of $\mathrm{RB} 1$ expression [abs $(z$-score $(\mathrm{RB} 1$ expression $)>1$ ].}

Reporting summary. Further information on research design is available in the Nature Research Reporting Summary linked to this article.

\section{Data availability}

The RNA expression and proteomics data referenced during the study are available in a public repository from the website: http://neellab.github.io/bfg/. All the other data supporting the findings of this study are available within the article and its supplementary information files. Data used to generate the figures are available in the Source Data file provided with this paper. Source data are provided with this paper.

\section{Code availability}

Code to reproduce the bioinformatics analyses and their related data is available at https://github.com/bhklab/TNBC_BAY-876.

Received: 8 August 2019; Accepted: 28 July 2020;

Published online: 21 August 2020

\section{References}

1. Torre, L. A. et al. Global cancer statistics, 2012. CA Cancer J. Clin. 65, 87-108 (2015).

2. Collignon, J., Lousberg, L., Schroeder, H. \& Jerusalem, G. Triple-negative breast cancer: treatment challenges and solutions. Breast Cancer 8, 93-107 (2016).

3. Morante, Z. et al. Post-recurrence survival in triple negative breast cancer. $J$. Clin. Orthod. 36, e13120-e13120 (2018).

4. Lee, A. \& Djamgoz, M. B. A. Triple negative breast cancer: emerging therapeutic modalities and novel combination therapies. Cancer Treat. Rev. 62, 110-122 (2018).

5. Bianchini, G., Balko, J. M., Mayer, I. A., Sanders, M. E. \& Gianni, L. Triplenegative breast cancer: challenges and opportunities of a heterogeneous disease. Nat. Rev. Clin. Oncol. 13, 674-690 (2016).

6. Kim, C. et al. Chemoresistance evolution in triple-negative breast cancer delineated by single-cell sequencing. Cell 173, 879-893.e13 (2018).

7. Martinez-Outschoorn, U. E., Peiris-Pagés, M., Pestell, R. G., Sotgia, F. \& Lisanti, M. P. Cancer metabolism: a therapeutic perspective. Nat. Rev. Clin. Oncol. 14, 113 (2017).
8. Chen, X.-S., Li, L.-Y., Guan, Y.-D., Yang, J.-M. \& Cheng, Y. Anticancer strategies based on the metabolic profile of tumor cells: therapeutic targeting of the Warburg effect. Acta Pharmacol. Sin. 37, 1013-1019 (2016).

9. Warburg, O. The metabolism of carcinoma cells. J. Cancer Res. 9, 148-163 (1925).

10. Pelicano, H. et al. Mitochondrial dysfunction in some triple-negative breast cancer cell lines: role of mTOR pathway and therapeutic potential. Breast Cancer Res. 16, 434 (2014).

11. Alluri, P. \& Newman, L. A. Basal-like and triple-negative breast cancers: searching for positives among many negatives. Surg. Oncol. Clin. N. Am. 23, 567-577 (2014).

12. Hussein, Y. R. et al. Glut-1 expression correlates with basal-like breast cancer. Transl. Oncol. 4, 321-327 (2011).

13. Chan, D. A. et al. Targeting GLUT1 and the Warburg effect in renal cell carcinoma by chemical synthetic lethality. Sci. Transl. Med. 3, $94 \mathrm{ra} 70$ (2011).

14. Ma, Y. et al. Ovarian cancer relies on glucose transporter 1 to fuel glycolysis and growth: anti-tumor activity of BAY-876. Cancers 11, 33 (2019).

15. Liu, Y. et al. A small-molecule inhibitor of glucose transporter 1 downregulates glycolysis, induces cell-cycle arrest, and inhibits cancer cell growth in vitro and in vivo. Mol. Cancer Ther. 11, 1672-1682 (2012).

16. Shriwas, P. et al. Abstract 2799: new-generation glucose transporter inhibitors targeting non-small cell lung cancer and triple-negative breast cancer. Cancer Res. 78, 2799-2799 (2018)

17. Oh, S., Kim, H., Nam, K. \& Shin, I. Glut1 promotes cell proliferation, migration and invasion by regulating epidermal growth factor receptor and integrin signaling in triple-negative breast cancer cells. BMB Rep. 50, 132-137 (2017).

18. Gandhi, N. \& Das, G. M. Metabolic reprogramming in breast cancer and its therapeutic implications. Cells 8, 89 (2019).

19. $\mathrm{Li}, \mathrm{H}$. et al. The landscape of cancer cell line metabolism. Nat. Med. 25, 850-860 (2019).

20. Levine, A. J. \& Puzio-Kuter, A. M. The control of the metabolic switch in cancers by oncogenes and tumor suppressor genes. Science 330, 1340-1344 (2010).

21. Nagarajan, A., Malvi, P. \& Wajapeyee, N. Oncogene-directed alterations in cancer cell metabolism. Trends Cancer Res. 2, 365-377 (2016).

22. Dai, C. et al. Metabolomics of oncogene-specific metabolic reprogramming during breast cancer. Cancer Metab. 6, 5 (2018).

23. Cancer Genome Atlas Network. Comprehensive molecular portraits of human breast tumours. Nature 490, 61-70 (2012).

24. Curtis, C. et al. The genomic and transcriptomic architecture of 2,000 breast tumours reveals novel subgroups. Nature 486, 346-352 (2012)

25. Parker, J. S. et al. Supervised risk predictor of breast cancer based on intrinsic subtypes. J. Clin. Oncol. 27, 1160-1167 (2009).

26. Chen, F., Chandrashekar, D. S., Varambally, S. \& Creighton, C. J. Pan-cancer molecular subtypes revealed by mass-spectrometry-based proteomic characterization of more than 500 human cancers. Nat. Commun. 10, 5679 (2019).

27. Bergqvist, F. et al. Anti-inflammatory properties of chemical probes in human whole blood: focus on prostaglandin E2 production. Front. Pharmacol. 11, 613 (2020).

28. Michealraj, K. A. et al. Metabolic regulation of the epigenome drives lethal infantile ependymoma. Cell 181, 1329-1345.e24 (2020).

29. Smolková, K. et al. Mitochondrial bioenergetic adaptations of breast cancer cells to aglycemia and hypoxia. J. Bioenerg. Biomembr. 42, 55-67 (2010).

30. Rossignol, R. et al. Energy substrate modulates mitochondrial structure and oxidative capacity in cancer cells. Cancer Res. 64, 985-993 (2004).

31. Jose, C., Bellance, N. \& Rossignol, R. Choosing between glycolysis and oxidative phosphorylation: a tumor's dilemma? Biochim. Biophys. Acta 1807, 552-561 (2011)

32. Krzeslak, A. et al. Expression of GLUT1 and GLUT3 glucose transporters in endometrial and breast cancers. Pathol. Oncol. Res. 18, 721-728 (2012).

33. Hamanaka, R. B. \& Chandel, N. S. Targeting glucose metabolism for cancer therapy. J. Exp. Med. 209, 211-215 (2012)

34. Marcotte, R. et al. Functional genomic landscape of human breast cancer drivers, vulnerabilities, and resistance. Cell 164, 293-309 (2016).

35. Li, J. et al. Characterization of human cancer cell lines by reverse-phase protein arrays. Cancer Cell 31, 225-239 (2017).

36. Dick, F. A., Goodrich, D. W., Sage, J. \& Dyson, N. J. Non-canonical functions of the RB protein in cancer. Nat. Rev. Cancer 18, 442-451 (2018).

37. Chen, H.-Z., Tsai, S.-Y. \& Leone, G. Emerging roles of E2Fs in cancer: an exit from cell cycle control. Nat. Rev. Cancer 9, 785-797 (2009).

38. Zacksenhaus, E. et al. Mitochondrial OXPHOS induced by RB1 deficiency in breast cancer: implications for anabolic metabolism, stemness, and metastasis. Trends Cancer Res. 3, 768-779 (2017).

39. Penault-Llorca, F. \& Radosevic-Robin, N. Ki67 assessment in breast cancer: an update. Pathology 49, 166-171 (2017).

40. Rizzardi, A. E. et al. Quantitative comparison of immunohistochemical staining measured by digital image analysis versus pathologist visual scoring. Diagn. Pathol. 7, 42 (2012). 
41. Fedchenko, N. \& Reifenrath, J. Different approaches for interpretation and reporting of immunohistochemistry analysis results in the bone tissue-a review. Diagn. Pathol. 9, 1-12 (2014).

42. Beatty, A. et al. Metabolite profiling reveals the glutathione biosynthetic pathway as a therapeutic target in triple-negative breast cancer. Mol. Cancer Ther. 17, 264-275 (2018).

43. Cheung, A. et al. Anti-folate receptor alpha-directed antibody therapies restrict the growth of triple-negative breast cancer. Clin. Cancer Res. 24, 5098-5111 (2018).

44. Camarda, R. et al. Inhibition of fatty acid oxidation as a therapy for MYC-overexpressing triple-negative breast cancer. Nat. Med. 22, 427-432 (2016).

45. Park, J. H. et al. Fatty acid oxidation-driven src links mitochondrial energy reprogramming and oncogenic properties in triple-negative breast cancer. Cell Rep. 14, 2154-2165 (2016).

46. Hay, N. Reprogramming glucose metabolism in cancer: can it be exploited for cancer therapy? Nat. Rev. Cancer 16, 635-649 (2016).

47. Sieow, J. L., Gun, S. Y. \& Wong, S. C. The sweet surrender: how myeloid cell metabolic plasticity shapes the tumor microenvironment. Front. Cell Dev. Biol. 6, 168 (2018).

48. Asgari, Y., Zabihinpour, Z., Salehzadeh-Yazdi, A., Schreiber, F. \& MasoudiNejad, A. Alterations in cancer cell metabolism: the Warburg effect and metabolic adaptation. Genomics 105, 275-281 (2015).

49. Lehuédé, C., Dupuy, F., Rabinovitch, R., Jones, R. G. \& Siegel, P. M. Metabolic plasticity as a determinant of tumor growth and metastasis. Cancer Res. 76, 5201-5208 (2016).

50. Lucantoni, F., Dussmann, H. \& Prehn, J. H. M. Metabolic targeting of breast cancer cells with the 2-deoxy-D-glucose and the mitochondrial bioenergetics inhibitor MDIVI-1. Front. Cell Dev. Biol. 6, 113 (2018).

51. Elstrom, R. L. et al. Akt stimulates aerobic glycolysis in cancer cells. Cancer Res. 64, 3892-3899 (2004).

52. Chen, C.-Y., Chen, J., He, L. \& Stiles, B. L. PTEN: Tumor Suppressor and Metabolic Regulator. Front. Endocrinol. 9, 338 (2018).

53. Camarda, R., Williams, J. \& Goga, A. In vivo Reprogramming of Cancer Metabolism by MYC. Front. Cell Dev. Biol. 5, 35 (2017).

54. Itahana, Y. \& Itahana, K. Emerging Roles of p53 family members in glucose metabolism. Int. J. Mol. Sci. 19, 776 (2018).

55. Jones, R. A. et al. RB1 deficiency in triple-negative breast cancer induces mitochondrial protein translation. J. Clin. Invest. 126, 3739-3757 (2016).

56. Nicolay, B. N. \& Dyson, N. J. The multiple connections between pRB and cell metabolism. Curr. Opin. Cell Biol. 25, 735-740 (2013).

57. Clem, B. et al. Loss of the retinoblastoma protein modulates glucose metabolism in lung cancer. FASEB J. 33, 652.4-652.4 (2019).

58. Nicolay, B. N. et al. Metabolic analysis of the loss of Rb1 in vivo. Cancer Metab. 2, O4 (2014).

59. Hirpara, J. et al. Metabolic reprogramming of oncogene-addicted cancer cells to OXPHOS as a mechanism of drug resistance. Redox Biol. 25, 101076 (2018).

60. Deblois, G. et al. Epigenetic switch-induced viral mimicry evasion in chemotherapy resistant breast cancer. Cancer Discov. https://doi.org/10.1158/ 2159-8290.CD-19-1493 (2020).

61. Patel, J. M. et al. Retinoblastoma $(\mathrm{Rb})$ protein expression in triple-negative breast cancer. J. Clin. Orthod. 35, 1097-1097 (2017).

62. Vivian, J. et al. Toil enables reproducible, open source, big biomedical data analyses. Nat. Biotechnol. 35, 314-316 (2017).

63. Gendoo, D. M. A. et al. Genefu: an R/Bioconductor package for computation of gene expression-based signatures in breast cancer. Bioinformatics 32, 1097-1099 (2016).

64. Hänzelmann, S., Castelo, R. \& Guinney, J. GSVA: gene set variation analysis for microarray and RNA-seq data. BMC Bioinforma. 14, 7 (2013).

65. Liberzon, A. et al. The Molecular Signatures Database (MSigDB) hallmark gene set collection. Cell Syst. 1, 417-425 (2015).

66. Chapman, P. J. et al. IncucyteDRC: an R package for the dose response analysis of live cell imaging data. F1000Res. 5, 962 (2016).

67. Smirnov, P. et al. PharmacoGx: an R package for analysis of large pharmacogenomic datasets. Bioinformatics 32, 1244-1246 (2016).

68. Sergushichev, A. An algorithm for fast preranked gene set enrichment analysis using cumulative statistic calculation. Preprint at https://doi.org/10.1101/ 060012 .

69. Sachs, N. et al. A living biobank of breast cancer organoids captures disease heterogeneity. Cell 172, 373-386.e10 (2018).

70. Li, J. et al. TCPA: a resource for cancer functional proteomics data. Nat. Methods 10, 1046-1047 (2013).

\section{Acknowledgements}

This study was conducted with the support of the Terry Fox Research Institute (New Frontiers Research Program PPG-1064, to D.W.C., B.H.-K., M.L., and C.H.A.), Canadian Cancer Research Society and the Ontario Institute for Cancer Research through funding provided by the Government of Ontario and the SGC. This work was also supported by the Canadian Institute for Health Research (CIHR project grant: Funding Reference Number 136963 to M.L., 363288 to B.H.-K., CIHR FDN154328 to C.H.A. and a CEEHRC team grant: Funding Reference Number 158225 to M.L. and C.H.A.), the Princess Margaret Cancer Foundation (M.L.) and the Gattuso-Slaight Personalized Cancer Medicine Fund at Princess Margaret Cancer Center (B.H.-K.). We acknowledge the Princess Margaret Bioinformatics group for providing the infrastructure assisting us with analysis presented here. Development of PDXDTO-B64 and PDXDTO-B81 was done in collaboration with the Princess Margaret Living Biobank. We thank the patients who provided tissue, the MUHC breast surgeons and pathologists, Virginie Pilon and Anie Monast for assistance with animal studies. We also appreciate the explant culture training provided by Yi Yin of Ganesh V. Raj's laboratory of UT Southwestern, in Dallas, TX, USA. G.D. is a recipient of fellowships from the CIHR, of the Fonds de Recherche en Santé du Québec (FRSQ) doctoral research award and of a Cancer Research Society Next-Generation of Scientists transition award. M.L. holds an Investigator Award from the Ontario Institute for Cancer Research and the Bernard and Francine Dorval Award for Excellence from the Canadian Cancer Society. The SGC is a registered charity (number 1097737) that receives funds from AbbVie, Bayer Pharma AG, Boehringer Ingelheim, Canada Foundation for Innovation, Eshelman Institute for Innovation, Genome Canada through the Ontario Genomics Institute [OGI-055], Innovative Medicines Initiative (EU/EFPIA) [ULTRA-DD grant no. 115766], Janssen, Merck KGaA, Darmstadt, Germany, MSD, Novartis Pharma AG, Pfizer, São Paulo Research FoundationFAPESP, Takeda, and Wellcome.

\section{Author contributions}

Q.W. designed and conducted the bulk of the experiments. W.B. implemented the bulk of the computational and statistical approaches. G.D. contributed to shaping the research and revised the manuscript for important intellectual content. G.D., A.-M.F., H.K., and J.C. conducted the PDXTOs assays. J.H. provided samples for PDXTEs. A.-M.F. performed TCGA analysis. T.D.M. helped histopathological image scanning. S.J.D. contributed to interpreting histopathological results of the tumor explant samples. S.D., E.L.M, N.D.A., W.J.C., S.Z. R.D., D.N. and LX. ZH. contributed to cell growing, immunoblotting, knockdown, seahorse assays. H.M. contributed to profile proteomics data. S.C. contributed to explant work. P.P., B.G.W., P.B.D. M.P., D.W.C., B.H.K., M.L. and C.H.A. supervised the research. M.L. and C.H.A. oversaw the project. Figures were designed and prepared by Q.W. and W.B. The manuscript was written by Q.W., M.L. and C.H.A. with assistance from all authors. All authors reviewed the final version of the manuscript and agreed with its content and submission.

\section{Competing interests}

The authors declare no competing interests.

\section{Additional information}

Supplementary information is available for this paper at https://doi.org/10.1038/s41467 020-18020-8.

Correspondence and requests for materials should be addressed to M.L. or C.H.A.

Peer review information Nature Communications thanks Tuuli Kaambre and the other, anonymous, reviewer(s) for their contribution to the peer review of this work.

Reprints and permission information is available at http://www.nature.com/reprints

Publisher's note Springer Nature remains neutral with regard to jurisdictional claims in published maps and institutional affiliations.

Open Access This article is licensed under a Creative Commons Attribution 4.0 International License, which permits use, sharing, adaptation, distribution and reproduction in any medium or format, as long as you give appropriate credit to the original author(s) and the source, provide a link to the Creative Commons license, and indicate if changes were made. The images or other third party material in this article are included in the article's Creative Commons license, unless indicated otherwise in a credit line to the material. If material is not included in the article's Creative Commons license and your intended use is not permitted by statutory regulation or exceeds the permitted use, you will need to obtain permission directly from the copyright holder. To view a copy of this license, visit http://creativecommons.org/licenses/by/4.0/.

(c) The Author(s) 2020 